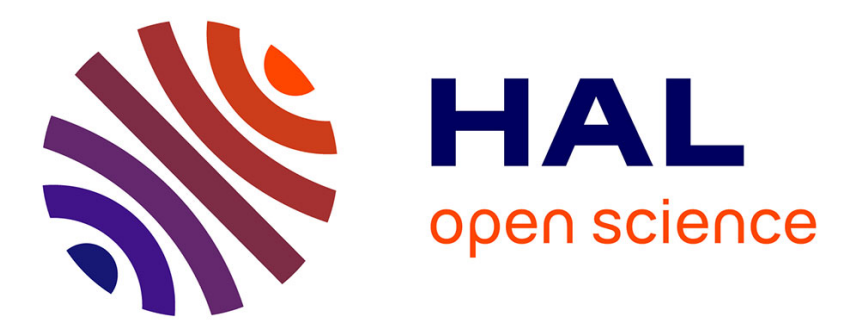

\title{
Seismic attenuation from recordings of ambient noise
}

Cornelis Weemstra, Lapo Boschi, Alexander Goertz, Brad Artman

\section{To cite this version:}

Cornelis Weemstra, Lapo Boschi, Alexander Goertz, Brad Artman. Seismic attenuation from recordings of ambient noise. Geophysics, 2013, 78 (1), pp.Q1-Q14. 10.1190/geo2012-0132.1 . hal-00828756

\section{HAL Id: hal-00828756 \\ https://hal.science/hal-00828756}

Submitted on 31 May 2013

HAL is a multi-disciplinary open access archive for the deposit and dissemination of scientific research documents, whether they are published or not. The documents may come from teaching and research institutions in France or abroad, or from public or private research centers.
L'archive ouverte pluridisciplinaire HAL, est destinée au dépôt et à la diffusion de documents scientifiques de niveau recherche, publiés ou non, émanant des établissements d'enseignement et de recherche français ou étrangers, des laboratoires publics ou privés. 


\title{
Seismic attenuation from
}

\section{recordings of ambient noise}

\author{
Cornelis Weemstra* ${ }^{* \dagger}$ Lapo Boschi ${ }^{\ddagger}$, Alexander Goertz ${ }^{\S}$ and Brad Artman* \\ * Spectraseis, Denver, Colorado, U.S.A. \\ ${ }^{\dagger}$ Institute of Geophysics, ETH, Sonneggstrasse 5, 8092 Zürich, Switzerland \\ $\ddagger$ Institute of Theoretical Physics, University of Zürich, Winterthurerstr. 190, 8057 \\ Zürich, Switzerland \\ $\S$ formerly Spectraseis AG, now at Swiss Seismological Service, Sonneggstrasse 5, 8092 \\ Zürich, Switzerland
}

(September 7, 2012)

Running head: attenuation from ambient noise

\begin{abstract}
We apply seismic interferometry to data from an OBS survey offshore Norway and show that ambient seismic noise can be used to constrain subsurface attenuation on a reservoir scale. By crosscorrelating only a few days of recordings by broad-band ocean bottom seismometers we are able to retrieve empirical Green's Functions (EGFs) associated with surface waves in the frequency range between 0.2 and $0.6 \mathrm{~Hz}$ and acoustic waves traveling through the seawater between 1.0 and $2.5 \mathrm{~Hz}$. We show that the decay of these surface waves cannot be explained by geometrical spreading alone and requires an additional loss of energy with distance. We quantify this observed attenuation in the frequency domain using a modified
\end{abstract}


Bessel function to describe the cross-spectrum in a stationary field. We average crossspectra of equally spaced station couples and sort these azimuthally averaged cross-spectra with distance. We then obtain frequency-dependent estimates of attenuation by minimizing the misfit of the real parts to a damped Bessel function. The resulting quality factors as function of frequency are indicative of the depth variation of attenuation and correlate with the geology in the survey area. 


\section{INTRODUCTION}

Passive seismic interferometry involves the crosscorrelation of ambient seismic noise recordings. The idea stems from the derivation of Claerbout (1968), who shows that the autocorrelation of the transmission response of a seismic noise source in the subsurface below a horizontally layered medium yields the reflection response of these horizontal reflectors plus its time-reversed version. He later made an analogous conjecture for the 3-D Earth (Rickett and Claerbout, 1999). In the eighties and nineties of the last century, several attempts have been made to retrieve this impulse response from crosscorrelation of real data, with the first convincing proof of concept produced by solar seismology (Duvall et al., 1993). The first successful application to acoustics, including a detailed proof of the theory, can be attributed to Lobkis and Weaver (2001). Other theoretical derivations are given, e.g., by Derode et al. (2003) and Snieder (2004) and, using a reciprocity theorem, by Wapenaar (2004). The first successful application to the solid Earth is due to Campillo and Paul (2003), who used seismic coda.

Shapiro and Campillo (2004) built on the work of Campillo and Paul (2003), showing that broadband Rayleigh waves emerge not only from the coda of earthquakes, but also by simply crosscorrelating ambient seismic noise recordings. These surface waves can be used for velocity inversion on a continental scale (Shapiro et al., 2005; Yang et al., 2007) as well as on a local scale (Brenguier et al., 2007; Bussat and Kugler, 2009). An elegant derivation of the underlying interferometric theory for surface waves is given by Tsai (2009). It has been shown that under certain circumstances and for specific locations and bandwidths, also body waves can be retrieved from the ambient seismic wavefield (e.g. Roux et al., 2005; Draganov et al., 2007; Zhang et al., 2009; Poli et al., 2012; Goertz et al., 2012). 
Ambient noise sources strongly vary with location and frequency. High levels of seismic noise are observed worldwide in the the frequency band from 0.05 to about $1 \mathrm{~Hz}$ (Peterson, 1993). The main peak within this bandwidth is the microseism peak. Microseisms are caused by ocean wave energy coupling into the solid earth. Typically, two types of microseisms, dubbed "primary" and "secondary", are observed at seismic stations. While the primary (or "single frequency") microseisms have the same frequency as the ocean waves that generate them, i.e. $\sim 0.08 \mathrm{~Hz}$, secondary (or "double frequency") microseisms are observed at twice this frequency. Explanations for the origin of microseisms were first given by Longuet-Higgins (1950) and Hasselmann (1963), but see, e.g. Stehly et al. (2006); Yang and Ritzwoller (2008); Landés et al. (2010) for an updated discussion.

Recently, several researchers have focused on estimating attenuation based on interferometric measurements of surface-wave empirical Green's functions (EGFs) made on regionalscale ( 100-1000 km) seismic arrays (Prieto et al., 2009; Lin et al., 2011; Lawrence and Prieto, 2011). We show here that the methodologies developed in those studies can be applied equally well at the local $(\sim 10-100 \mathrm{~km})$ scale. Robust estimates of seismic attenuation at this small scale are needed for several reasons. First, surface waves traveling through sedimentary basins, whose seismic velocity is low, are amplified by virtue of energy conservation, but the low quality factor that also characterizes sediments counters this effect, reducing amplitudes again. Neglecting this phenomenon may lead to overestimation of ground-motion predictions. Second, hydrocarbon reservoirs exhibit an abnormally strong attenuation contrast (Chapman et al., 2006), and measuring attenuation based on the observed ambient seismic wavefield might thus help us to discriminate a reservoir's fluid content. It should be noted that, as a consequence, the inversion of the ambient seismic wavefield for attenuation may provide a means to image hydrocarbon reservoirs. Finally, a good S-wave velocity and 
attenuation model of the shallow subsurface would help to improve S-wave static corrections needed in oil and gas exploration.

Prieto et al. (2009) show how one-dimensional depth profiles of phase velocity and quality factor can be effectively derived from stacked crosscorrelations of ambient noise. This approach is extended to the case of three-dimensional velocity/quality factor heterogeneity by Lawrence and Prieto (2011), who recover phase velocity and attenuation coefficient maps at periods of 8-32 s for the western United States. Lin et al. (2011) follow a different approach, evaluating amplitude-decay of time-domain crosscorrelations.

We adapt both the approaches of Prieto et al. (2009) and Lin et al. (2011) to the reservoir-scale inverse problem associated with seismic recordings collected by a broadband ocean-bottom survey in the North Sea, covering an area of $\sim 220 \mathrm{~km}^{2}$ and with average interstation spacing of $\sim 500 \mathrm{~m}$. This demands several changes, described below, to the data preprocessing and inversion procedures of Prieto et al. (2009) and Lin et al. (2011). We do not invert for velocity and attenuation structure as a function of depth, but merely compare the frequency-dependent velocity and attenuation to the depth-dependent geology, finding an interesting correlation.

We first identify an amplitude decay of the EGFs that cannot be associated with geometrical spreading and hence represents the effect of energy dissipation and scattering. Second, we provide a reliable estimate of this excess decay in the study area based on the frequency-domain results of our experiment. Because of the short duration of the survey, and the difficulties intrinsic to operating ocean-bottom instrumentation, however, available data do not allow to robustly constrain lateral variations in attenuation.

In the following, we first summarize the theory of seismic noise interferometry and its 
relation to the normalized spatial autocorrelation (SPAC) of Aki (1957). We point out the necessary assumptions and approximations and consequent limitations of our formulation. We then describe the database available to us, formulate the associated inverse problem, and finally discuss its solution and implications.

\section{THEORY}

An isotropic wavefield is a prerequisite for obtaining symmetric EGFs (Snieder et al., 2007). Such a wavefield can be generated by a homogeneous distribution of uncorrelated stochastic sources surrounding the array (e.g. Larose et al., 2006; Wapenaar et al., 2010), or by multiple scattering among heterogeneities in a complex medium (Campillo and Paul, 2003). The relation between the SPAC applied to a seismic wavefield (Aki, 1957) on one hand and timedomain cross correlation of ambient seismic noise on the other hand, pointed out by Yokoi and Margaryan (2008) and shown explicitly by Tsai and Moschetti (2010), is the backbone of the method applied here because it justifies azimuthal averaging of crosscorrelations in a non-isotropic seismic wavefield.

Given the recordings $u\left(\boldsymbol{x}_{\mathbf{1}}\right)$ and $u\left(\boldsymbol{x}_{\mathbf{2}}\right)$, captured at surface locations $\boldsymbol{x}_{\mathbf{1}}$ and $\boldsymbol{x}_{\mathbf{2}}$, the normalized time-domain cross-correlation $C_{x_{1} x_{2}}$ is defined

$$
C_{x_{1} x_{2}}(t) \equiv \frac{1}{2 T} \int_{-T}^{T} u\left(\boldsymbol{x}_{1}, \tau\right) u\left(\boldsymbol{x}_{\mathbf{2}}, \tau+t\right) d \tau
$$

where $t$ is time, $\tau$ is integration time and correlation time is given by $2 T$.

In general, the SPAC is used to find similarities in a spatial field by evaluating, for different space shifts $\boldsymbol{\xi}$, the product of the field with a space shifted version of itself. This product is then integrated over, and normalized by the size of the field $A$, where the unit of $A$ depends on the dimension of the actual field, e.g. $m^{2}$ for area or $m^{3}$ for volume. 
The SPAC, as applied by Aki, similarly yields the "coherency" $\phi(\boldsymbol{\xi})$ of a time-dependent wavefield $u(\boldsymbol{x}, t)$ for a shift in space by $\boldsymbol{\xi}$ and is defined as

$$
\phi(\boldsymbol{\xi}, t) \equiv \frac{1}{A} \int_{A} u(\boldsymbol{x}, t) u(\boldsymbol{x}+\boldsymbol{\xi}, t) d \boldsymbol{x},
$$

where $\boldsymbol{x}$ denotes position and $A$ describes the area over which the wavefield is evaluated. The integration over, and normalization by $A$ in equation 2 averages the product in the integral over different $\boldsymbol{x}$. For that reason, $\phi(\boldsymbol{\xi})$ is also called the spatially averaged coherency (Asten, 2006).

Aki (1957) assumes a wavefield that is stationary in both time and space. Here, "stationary" means that the amplitude is described by a stochast, whose joint probability distribution does not change when shifted in time or space. It follows that for such a wavefield the integral in equation 2 can be replaced by a time integral (Aki, 1957):

$$
\phi(\boldsymbol{\xi}) \equiv \frac{1}{2 T} \int_{-T}^{T} u(\boldsymbol{x}, t) u(\boldsymbol{x}+\boldsymbol{\xi}, t) d t .
$$

Important requirement here is that $T$ needs to be sufficiently long to obtain an approximately constant $\phi(\boldsymbol{\xi})$. The assumption of a stationary $u(\boldsymbol{x}, t)$, makes $\phi(\boldsymbol{\xi})$ independent of $\boldsymbol{x}$.

Following Aki (1957), an "average coherency" $\langle\phi\rangle$ can be introduced,

$$
\langle\phi(r)\rangle \equiv \frac{1}{2 \pi} \int_{|\boldsymbol{\xi}|=r} \phi(\boldsymbol{\xi}) d \boldsymbol{\xi}=\frac{1}{2 \pi} \int_{0}^{2 \pi} \phi(|\boldsymbol{\xi}|=r) d \theta
$$

with $|\boldsymbol{\xi}|=r$ the distance between two stations and $\theta$ their azimuth. After replacing $\phi(\boldsymbol{\xi})$ in equation 4 with its expression 3 , we obtain

$$
\langle\phi(r=|\boldsymbol{\xi}|)\rangle \equiv \frac{1}{4 \pi T} \int_{0}^{2 \pi} \int_{-T}^{T} u(\boldsymbol{x}, t) u(\boldsymbol{x}+\boldsymbol{\xi}, t) d t d \theta .
$$

It is important to note here that the average coherency is an average over both time and azimuth, while the coherency is only an average over time (see equation 3.) The averaging is 
completely transparent in the ideal case of noise sources distributed uniformly in azimuth: in this case, $\phi(\boldsymbol{\xi})=\langle\phi(r)\rangle$ if $|\boldsymbol{\xi}|=r$.

Tsai and Moschetti (2010) consider a single deterministic wave of frequency $\omega$ and solve the time integral in 3 assuming the correlation time $2 T$ is sufficiently long with respect to the period of the waves. They next combine (through a simple integral) multiple sources, assuming them to be uncorrelated such that the crosscorrelations of signals generated by different sources (the "cross-terms") cancel out. This assumption is equivalent to the assumption of stationarity by Aki (1957). Similar assumptions have been made by other authors (Lobkis and Weaver, 2001; Snieder, 2004; Wapenaar, 2004). Through this procedure, Tsai and Moschetti (2010) find analytically that

$$
\frac{\langle\phi(r, \omega)\rangle}{\phi(0, \omega)}=J_{0}\left(\frac{r \omega}{c(\omega)}\right)
$$

where $J_{0}$ denotes the $0^{\text {th }}$ order Bessel function of the first kind, and $c(\omega)$ is the wave velocity as function of angular frequency. This expression coincides with equation (42) of Aki (1957). The left-hand side of equation 6 is denoted $\langle\rho(r, \omega)\rangle \equiv\langle\phi(r, \omega)\rangle / \phi(0, \omega)$ and dubbed "averaged complex coherency". Note that this averaged complex coherency is normalized by the energy $(\phi(0, \omega))$, which will be independent of location and frequency for a stationary wavefield.

Tsai and Moschetti (2010) also show that the real part of the cross-correlation spectrum coincides with the SPAC. If we define the cross-spectrum $\hat{C}_{x_{1} x_{2}}(\omega) \equiv \mathbb{F}\left[C_{x_{1} x_{2}}(t)\right]$, where $\mathbb{F}$ is the Fourier-transform operator, we get,

$$
\Re\left[\hat{C}_{x_{1} x_{2}}(\omega)\right]=\phi(\boldsymbol{\xi}, \omega) .
$$

The operator $\Re[\ldots]$ maps its complex argument into its real part, $\boldsymbol{\xi}=\boldsymbol{x}_{\mathbf{1}}-\boldsymbol{x}_{\mathbf{2}}$. For a stationary wavefield, this crosscorrelation spectrum, similar to the SPAC in equation 3, 
will converge towards one value when averaged over time. Equation 7, together with equation 6 , implies that an isotropic source distribution results in $\Re\left[\hat{C}_{x_{1} x_{2}}(\omega)\right]$ coinciding with $J_{0}(|\boldsymbol{\xi}| \omega / c(\omega))$ multiplied by a constant related to the power at frequency $\omega$, while its imaginary part will be zero (Tsai and Moschetti, 2010).

Time-averaging of coherencies stabilizes the average coherency in the case of a stationary wavefield (Okada, 2003). We denote $E\left[\hat{C}_{x_{1} x_{2}}(\omega)\right]$ the ensemble average of $\hat{C}_{x_{1} x_{2}}(\omega)$ over different time-windows. Similar to the normalization of the average coherency by the time-averaged energy $\phi(0, \omega)$ to obtain the averaged complex coherency $\langle\rho(r, \omega)\rangle$, we can normalize $E\left[\hat{C}_{x_{1} x_{2}}(\omega)\right]$ with respect to the time-averaged power of the wavefield at the two stations at $\boldsymbol{x}_{\mathbf{1}}$ and $\boldsymbol{x}_{\mathbf{2}}$, which is approximated by $\sqrt{\left|u\left(\boldsymbol{x}_{\mathbf{1}}, \omega\right)\right|^{2}\left|u\left(\boldsymbol{x}_{\mathbf{2}}, \omega\right)\right|^{2}}$. If the source distribution is anisotropic, one can average both sides of equation 7 over azimuth and using equation 6 we get:

$$
\left\langle\frac{\Re\left\{E\left[\hat{C}_{x_{1} x_{2}}(\omega)\right]\right\}}{\sqrt{E\left[\left|u\left(\boldsymbol{x}_{\mathbf{1}}, \omega\right)\right|^{2}\right] E\left[\left|u\left(\boldsymbol{x}_{\mathbf{2}}, \omega\right)\right|^{2}\right]}}\right\rangle=\langle\rho(r, \omega)\rangle=J_{0}\left(\frac{r \omega}{c(\omega)}\right) .
$$

Azimuthal averaging is, just as for Aki's SPAC, over all $\boldsymbol{x}_{\mathbf{1}}$ and $\boldsymbol{x}_{\mathbf{2}}$ for which $\left|\boldsymbol{x}_{\boldsymbol{1}}-\boldsymbol{x}_{\mathbf{2}}\right|=r$.

If the source distribution changes sufficiently over a certain timespan, averaging over this timespan is similar to averaging over azimuth for a fixed source distribution (Chávez-García et al., 2005). This means that if one has data for a 'sufficient' amount of time, one can set up an inverse problem for a single station couple, i.e. fixed $\boldsymbol{\xi}$, with the phase-velocity dispersion $c(\omega)$ as unknown. Ekström et al. (2009) implement such an inverse problem by equating the known zeros of $J_{0}(|\boldsymbol{\xi}| \omega / c(\omega))$ to the observed zero crossings of $\Re\left[\hat{C}_{x_{1} x_{2}}(\omega)\right]$.

It should be noted that throughout this formulation, azimuthal averaging would not be needed in the ideal case of a wavefield generated by an azimuthally uniform distribution of sources. In the real world, averaging is needed to mimic an isotropic wavefield, combining 
a random set of ballistic fields recorded at different times and/or locations.

The way of calculating the averaged complex coherency in equation 8 is the de facto standard practice in the SPAC-community (Okada, 2003), that is, the cross-spectra are first ensemble-averaged and subsequently normalized by the average power spectra (Okada, 2003). Tsai (2011, eq. 21) evaluates essentially the same expression, deriving the coherency in the limit of infinite time for fixed sources with random phases, and hence assuming a stationary wavefield. In the case of Tsai (2011), this assumption is required to justify neglecting the aforementioned cross-terms. While this assumption is theoretically correct, it is somewhat unrealistic in the application to real seismic data as these are in general recorded over a wavefield that is subject to continuous change (see e.g. Stehly et al., 2006; Olofsson, 2010).

Recent successful studies using surface waves obtained from ambient noise to constrain subsurface attenuation use a modified expression of equation 8 (Prieto et al., 2009; Lawrence and Prieto, 2011). Latter authors "whiten" the recordings prior to crosscorrelation and obtain the "whitened complex coherency":

$$
\gamma(r, \omega) \equiv\left\langle E\left[\frac{\Re\left[\hat{C}_{x_{1} x_{2}}(\omega)\right]}{\left|u\left(\boldsymbol{x}_{1}, \omega\right)\right|\left|u\left(\boldsymbol{x}_{\mathbf{2}}, \omega\right)\right|}\right]\right\rangle .
$$

Whitening is a commonly used tool to increase signal-to-noise ratios of signal from crosscorrelations of ambient seismic noise (Bensen et al., 2007; Brenguier et al., 2007; Seats et al., 2012). Whitened spectra are relieved of contamination by resonance peaks in the spectra (Bensen et al., 2007; Brenguier et al., 2007) and allow for the wavefield to be non-stationary between individual time-windows. The whitened complex coherency will be a scaled version of the Bessel function:

$$
\gamma(r, \omega)=A(\omega) J_{0}\left(\frac{r \omega}{c(\omega)}\right)
$$


The proportionality factor $A(\omega)$, with value between 0 and 1 , needs to be introduced to account for the cross-terms and is due to the difference in normalization between equation 8 and 9. The two amplitudes in the denominator of equation 9, i.e. $\left|u\left(\boldsymbol{x}_{\mathbf{1}}, \omega\right)\right|$ and $\left|u\left(\boldsymbol{x}_{\mathbf{2}}, \omega\right)\right|$, are not individually ensemble-averaged and hence have a high probability of being different valued for different time-windows. This is due to the random phase of the cross-terms (Tsai, 2011, eq. 18). Conversely, the two ensemble-averaged amplitudes in the denominator of equation 8 will be close to equal valued. This important consideration could potentially be used to obtain an ideal period of time over which to apply equation 8 after which expression 9 could be used to average over these periods; this is beyond the scope of this work however. We acquiesce in using equations 9 and 10 in the knowledge that each interstation distance is equally affected by this phenomenon, i.e. $A(\omega)$ is independent of $r$.

Equation 10 clearly does not account for the effects of attenuation. Prieto et al. (2009) propose to include attenuation through multiplication by an exponential factor $e^{-\alpha(\omega) r}$,

$$
\gamma(r, \omega)=A(\omega) J_{0}\left(\frac{r \omega}{c(\omega)}\right) e^{-\alpha(\omega) r}
$$

The "attenuation coefficient" $\alpha(\omega)$ accommodates a more rapid decrease of the Bessel function with interstation distance $r$, representing the effect of energy dissipation and scattering. $\alpha$ is related to the surface wave quality factor $Q$ by (Aki and Richards, 2002)

$$
\alpha(\omega)=\left(\frac{\omega}{2 Q U(\omega)}\right)
$$

where $U(\omega)$ is the group velocity. Given a series of azimuthally and temporally averaged complex coherencies for various interstation distances, a frequency-dependent estimate of $Q$ can be obtained. As surface waves of different frequencies sample different depths, the change of $Q$ with frequency can, to the first order, be associated with a change in material properties as a function of depth. The frequency dependent estimate of $Q$ is obtained by 
minimizing the error on the fit of the "damped" Bessel function to the real parts of the averaged complex coherencies.

Tsai (2011) shows that in the presence of attenuation, the source distribution determines how the coherency decays with distance. He shows for a number of specific source distributions how the real part of the coherency behaves as a function of distance for a laterally invariant $c(\omega)$ and $\alpha(\omega)$. If one evaluates the decay of the (azimuthally) averaged complex coherency however, only radial symmetric source distributions need to be considered, and the behavior of the coherency with distance is described by a Bessel function multiplied by a term decaying with distance. The rate of decay of this term depends on the distribution of sources away from the station couple, i.e. the radial distribution of sources (Tsai, 2011). Since our data consists of recordings of broad-band ocean-bottom seismometers (BBOBS) over a survey area offshore Norway, we assume a uniform radial distribution of noise sources with distance away from the center of the array. For such a distribution of sources, multiplication of the Bessel function with a decaying exponential term as in equation 11 is justified (Tsai, 2011).

\section{DATA}

We analyze a passive seismic data set acquired in April-May 2007, and consisting of broadband ocean-bottom recordings distributed over a $\sim 220 \mathrm{~km}^{2}$ survey area offshore Norway. The average water depth is $360 \mathrm{~m}$ and only deviates by a maximum of $\sim 9 \mathrm{~m}$ from this average. Figure 1 shows the configuration of the array and the duration of recording at each of the total of 117 locations. Stations did not all record synchronously: of the 16 available instruments, 14 were systematically redeployed at new locations after 1 to 2 days of recording, and only two recorded continuously for $>12$ days. 
[Figure 1 about here.]

The constant station spacing of $\sim 500 \mathrm{~m}$ gives rise to a very nonuniform distribution of the number of station couples and synchronous time windows with interstation distance, as shown by a histogram of synchronous 60 -s windows in Figure 2. A bin width of 100 $\mathrm{m}$ is used to select and sort the station couples with respect to interstation distance. The appearance of both distributions is similar and differences arise from different synchronous recording lengths for different station couples. Beyond $\sim 12000 \mathrm{~m}$ offset, the number of synchronous time windows decreases significantly.

Stations were all equipped with a broadband seismometer (Nanometrics Trillium 240) and a differential pressure gauge (DPG) built by the Scripps Institution of Oceanography (Cox et al., 1984). The instruments have a flat response to particle velocity between $240 \mathrm{~s}$ and $50 \mathrm{~Hz}$, and data are acquired with a sampling rate of $125 \mathrm{~Hz}$.

A thorough description of the noise characteristics above $\sim 3 \mathrm{~Hz}$ is given by Olofsson (2010), who finds that distinct noise of various origin can be found at frequencies above $\sim 3$ Hz. Some of these sources are anthropogenic (i.a. closely passing ships and a nearby seismic survey) and some are environmental (e.g. flagpole vibrations caused by water currents). All are outside our frequency range of interest.

[Figure 2 about here.]

Most ambient signal at frequencies below $\sim 3 \mathrm{~Hz}$ stems from infragravity waves and Scholte waves (Olofsson, 2010); Scholte waves are waves arising at a fluid-solid interface (Scholte, 1943). They have a particle motion similar to Rayleigh waves, but the velocities are generally lower due to interaction with the overlying water column. Scholte waves can be 
regarded as Rayleigh waves in the longer wavelength limit (Bohlen et al., 2004). Dispersion of the Scholte waves is caused by sediment layering and the finite water depth.

The single-frequency microseism peak is not obvious in the data, but the doublefrequency peak is clearly visible between 0.15 and $0.40 \mathrm{~Hz}$ (Olofsson, 2010; Bussat and Kugler, 2011) (see also Figure 7). Triangulation of frequency-wavenumber analysis results of two arrays in Europe indicate, at least in winter 1995-1996, that a region near the northNorwegian coast is a strong source of secondary microseisms (Friedrich et al., 1998). Two modes are distinguished in the crosscorrelations of the vertical component data. These modes can be found in the DPG-component crosscorrelations as well, but this component also shows a third mode due to water-guided waves as shown by Bussat and Kugler (2011). They were able to apply ambient-noise surface-wave tomography methods on fundamentalmode Scholte waves recorded on the DPG-component.

\section{PREPROCESSING}

Because of the continuously changing station configuration, the first step is to evaluate which stations are synchronously recording at which times. For each station couple, the obtained period of synchronous recording is then cut in time windows of 60 seconds. We cut neighboring time windows so that they overlap by as much as $75 \%$ of their total length. Seats et al. (2012) show that overlap helps refining EGFs, by avoiding loss of coherent signal traveling between a pair of stations, and this is particularly useful here as the temporal extent of the survey was very limited. We Fourier-transform individual time windows, and crosscorrelate in the frequency domain (i.e. multiply) the spectra of simultaneous signals associated with different stations. More specifically, we follow the following processing sequence for each time-window: 
1. Detrend the time-window.

2. Apply a cosine taper of $2.5 \%$ of the trace length.

3. Fourier transform the traces.

4. Whiten the amplitude spectra.

5. Multiply the spectrum of the first station with the complex conjugate of the spectrum of the second station, i.e. frequency-domain cross correlation.

We next stack individual cross-spectra to form an ensemble averaged cross-spectrum for each station couple. Time-domain EGFs are obtained by inverse-Fourier transforming the ensemble averaged cross-spectra. The ensemble averaged cross-spectra associated with the same interstation distance $r$ are stacked, i.e. the data are averaged with respect to azimuth. This way, any information about lateral structure across the array will be lost, but our database would hardly be able to resolve it anyway. The result is a measure of $\gamma(r, \omega)$.

Whitening of the amplitude spectra before crosscorrelation consists of setting the power of each frequency equal to 1 (see equation 9). This means that the time-domain amplitude information is destroyed, but phase information is retained. For our data this means that the higher frequencies are amplified with respect to the lower frequency content of the microseism's peak. The whitening procedure also removes power differences between recordings and hence near-receiver amplitude effects are removed this way.

Each individual coherency has the same weight in the ensemble averaging process of the whitened cross-spectra, because the denominator in equation 9 is the power associated with the individual time-windows at the two station locations. Note that this normalization is slightly different from the approach of Prieto et al. (2009); Lawrence and Prieto (2011) who 
smooth the two amplitude spectra before the cross-spectrum is divided by their product.

\section{ISOTROPY \& TIME-DOMAIN ATTENUATION}

[Figure 3 about here.]

The Green function gathers (GFG's) in Figure 3 show a clear moveout, with moveout velocity $\sim 500 \mathrm{~m} / \mathrm{s}$ for the lower frequency band (a), and $\sim 1500 \mathrm{~m} / \mathrm{s}$ for the 1.0 to $2.0 \mathrm{~Hz}$ band. The frequency bands of Figure 3 are chosen based on the slowness-frequency plots in Bussat and Kugler (2011). The 0.2 to $0.4 \mathrm{~Hz}$ band corresponds to the fundamental-mode Scholte waves, while we attribute the 1.0 to $2.0 \mathrm{~Hz}$ moveout to acoustic waves traveling through the water column just above the seabed: a group velocity of $\sim 1500 \mathrm{~m} / \mathrm{s}$ coincides with the speed of sound in water.

Figure 3 only includes crosscorrelations with relatively high signal-to-noise ratio (SNR). We define SNR empirically as the maximum absolute amplitude in the velocity wedge of interest $(350 \mathrm{~m} / \mathrm{s}$ to $750 \mathrm{~m} / \mathrm{s}$ for Scholte waves and 1000 to $3000 \mathrm{~m} / \mathrm{s}$ for water-guided waves) divided by the standard deviation of the noise windows. The noise windows are defined as the windows corresponding to higher and lower velocities than the velocity range of interest. A transitional margin outside of the velocity range of interest is employed, whose width depends on the frequency band of interest. Longer periodic crosscorrelations have longer margins between "signal window" and "noise window".

[Figure 4 about here.]

[Figure 5 about here.] 
Figure 4 summarizes the temporal change of the incident vertical-component wavefield for the frequency band between 0.20 and $0.40 \mathrm{~Hz}$ (i.e. fundamental-mode Scholte waves). The most symmetric EGF, observed on day 12, is shown in Figure 5 a while the EGF with causal and anti-causal segments of most different amplitude is observed on day 7 and shown in Figure 5 b. Circles in Figure 4 are plotted along station-couple back azimuth, and hence point towards the half plane where most energy is generated. In terms of source distributions, this can be either a small region with a high volume density of force or a broad region with only a slight increase in volume density of force. These two end-members are indistinguishable based on one EGF alone.

From Figure 4 one can in principle estimate the average direction of propagation, and relative strength of the ambient wavefield. This is in turn determined by a number of factors. First, the source distribution: note, e.g., that a fully isotropic wavefield would result in circles associated with station couples all falling in the center of the polar plot, independent of azimuth. Second, the change of this source distribution over time, because EGFs represent different periods of one day. Third, the array configuration. Fourth, the interstation distance, with the associated amplitude attenuation caused by geometrical spreading as well as attenuation due to the medium properties. Finally, the vertical and lateral structure of the subsurface can change the amount of decay from place to place through differences in attenuation. We define attenuation here as the the sum of "scattering" and "intrinsic" (i.e. absorption of energy) attenuation as we are unable to separate the two.

[Figure 6 about here.]

Our goal with this study is to try to isolate attenuation effects, and, if possible, the difference between attenuation due to geometrical spreading and attenuation due to medium 
properties, from all other factors. To evaluate whether this is possible we select two subsets of high-SNR ( $>4$ ) synchronous EGFs, each corresponding to stations lying along one straight line (at interstation distances $>3.2 \mathrm{~km}$ ). We plot in Figure 6 EGF amplitude as a function of interstation distance. Importantly, there is no temporal variation of the source distribution between stations of the same subset because all EGFs are based on the same period ( $>4$ hours) of recording. We next compare these EGF amplitudes to amplitude decay proportional to $a / \sqrt{r}$ caused by simple geometrical spreading (dashed lines) and amplitude decay proportional to $a / \sqrt{r} \times e^{-\alpha r}$ (solid lines). The latter model accounts for scattering and dissipation of energy of the ambient field. The constant $a$ and attenuation coefficient $\alpha$ are found by minimizing the L1-norm of the differences between datapoints and the $a / \sqrt{r} \times e^{-\alpha r}$ curves. Figure 6 clearly suggests that the EGFs amplitudes decay faster than with $\sqrt{r}$ and that an attenuating model explains the data better for both causal and anti-causal parts of all data subsets. We conclude that attenuation effects other than simple geometrical spreading can be inferred from the data, and proceed to evaluate them quantitatively.

\section{FREQUENCY-DOMAIN ATTENUATION}

Azimuthal averaging of cross-spectra associated with equally spaced station couples approximates a Bessel function (Prieto et al., 2009; Lawrence and Prieto, 2011; Tsai and Moschetti, 2010; Tsai, 2011). The quality of the approximation depends on the lateral variation of the subsurface velocity and attenuation structure, the spatial sampling of the wavefield and the length of the crosscorrelated time windows. The correlation time $T$ needs to be sufficiently long with respect to the period of the waves considered, otherwise a significant error will be introduced; The degree of stationarity of the wavefield trades off with the length of $T$ 
(Tsai and Moschetti, 2010). In our case $T \gg 1 / \omega$ for the 60 seconds windowing applied and frequencies evaluated here.

The whitened complex coherency could also decay faster than a Bessel function due to strong incoherent noise. In the presence of such noise, a constant value associated with the incoherent noise amplitude would be a fraction of both frequency-domain amplitudes in the denominator of equation 9 . We expect this to have little to no effect on our analysis for the considered frequencies, i.e. $\sim 0.20-2.20 \mathrm{~Hz}$. This is based on two observations. First, Ringler and Hutt (2010) show that the instrument-noise of the Nanometrics Trillium 240 is in the order of $\sim 15$ DB below the low-noise model of Peterson (1993). Second, we don't have any (incoherent) cultural noise sources within our array. Figure 7 gives example power spectral densities (PSDs) that are typical for our dataset. PSDs for two periods for stations P01 and B01 are presented where station B01 is denoted by the far most left dot in Figure 1. It is clear that the power of our recordings is significantly higher than that of the low-noise model for the frequency range considered (see also Olofsson, 2010).

We expect the power of the coherent noise at our survey site to be significantly higher than that of the new low-noise model. We base this on the observation that stations located close to the ocean or on oceanic islands in general show significantly higher (coherent) noise levels (McNamara and Buland, 2004; Peterson, 1993), while our instruments are even deployed at the bottom of the ocean.

[Figure 7 about here.]

Frequency-slowness analysis of the EGFs shows that two surface-wave modes are observed on the DPG component and vertical component: a fundamental mode and a firsthigher order mode (Bussat and Kugler, 2011). These modes depend locally on the subsurface 
elastic properties (e.g. Aki and Richards, 2002). Averaging over different couples, as done here, is meaningful so far as lateral changes of these properties are sufficiently smooth.

We average over interstation azimuths by binning interstation distances into bins with a width of $100 \mathrm{~m}$. Complex coherencies of station couples within each bin are averaged. whitened complex coherencies are attributed to the mean interstation distance of the averaged time windows. Bins representing less than 3 station couples or less than 6 hours of synchronous recordings are not taken into account. We evaluate two frequency bands: one for the vertical component and one for the DPG-component. The frequencies we evaluate for the vertical component $(0.20-0.40 \mathrm{~Hz})$ correspond to fundamental-mode Scholte waves (Bussat and Kugler, 2011). We assured ourselves that the upper bound of $0.4 \mathrm{~Hz}$ employed is not affected by the first-higher order mode Scholte waves. We analyze the acoustic mode of the DPG-component from 1.50 to $2.20 \mathrm{~Hz}$. The DPG-component analysis enables us to compare the obtained attenuation coefficients against values for the attenuation of seawater that can be found in the literature.

[Figure 8 about here.]

Figure 8 shows the azimuthally averaged real parts, i.e. $\gamma(r, \omega)$, for interstation distances with "sufficient" data coverage (bins that include $\geq 3$ station couples and $>6$ hours of synchronous recordings). The oscillating behavior of a Bessel function can clearly be observed for both the vertical and DPG-component. Mind that the color scale is different for the two plots. Especially the vertical component shows a decay with distance that is significantly stronger than that of a Bessel function. We determine this decay independently for each frequency by fitting the damped Bessel function defined in equation 11, introducing 
the misfit function

$$
M F(A, \alpha, c)=\sum_{i=1}^{N}\left|\gamma\left(r_{i}\right)-A J_{0}\left(\frac{r_{i} \omega}{c}\right) e^{-\alpha r_{i}}\right|
$$

based on the L1-norm, where $N$ is the number of bins that meet the requirements regarding data coverage and $A$ denotes the vertical offset at $r=0$ associated with the proportionality in equation 11. The L1-norm mitigates the effect of outliers due to limited azimuthal and/or temporal coverage in some bins (Lawrence and Prieto, 2011).

We conduct a three-dimensional grid search to find the values of attenuation $\alpha$, velocity $c$ and offset $A$ that minimize $M F$. For the vertical component, $c$ is sampled from 500 to 4000 $\mathrm{m} / \mathrm{s}$ and incremented by $2 \mathrm{~m} / \mathrm{s}, \alpha$ values are increased from 0 to $0.0002 \mathrm{~Np} / \mathrm{m}$ in 200 steps and $A$ is varied between 0 and 1 with an increment of 0.005 . On the DPG-component, $c$ varies between 1000 and $2500 \mathrm{~m} / \mathrm{s}$ and $A$ between 0 and 1 with the same increment, while $\alpha$ varies between 0 and $2 \times 10^{-4} \mathrm{~Np} / \mathrm{m}$ with an increment of $10^{-6} \mathrm{~Np} / \mathrm{m}$, as attenuation turns out to be significantly lower for acoustic-mode waves. Figure 9 and 10 show the damped Bessel functions $J_{0}\left(\frac{\omega r_{i}}{c}\right) e^{-\alpha r_{i}}$ associated with the values of $c, A$ and $\alpha$ that minimize $M F$ for the vertical (at $0.25 \mathrm{~Hz})$ and DPG $(2.00 \mathrm{~Hz})$ components, respectively.

[Figure 9 about here.]

[Figure 10 about here.]

From both Figure 9 and 10 it is clear that the behavior of $\gamma(r)$ is better explained by a damped than by a pure Bessel function. The improved fit is observed for all evaluated frequencies of both components $(0.20-0.40 \mathrm{~Hz}$ vertical-component band and 1.50-2.20 Hz band of the DPG component). Tsai (2011) shows that for a source distribution of only far 
field sources, amplitudes decay with $1 / I_{0}(\alpha r)$ instead of $1 / e^{\alpha r}$, where $I_{0}$ is the $0^{\text {th }}$ order modified Bessel function of the first kind. Explaining the slower decay of the data points with the wrong model, i.e. $1 / e^{\alpha r}$ instead of $1 / I_{0}(\alpha r)$, would result in a consistent underestimation of the data points for short interstation distances and a consistent overestimation of the data points for long interstation distances. On the other hand, in case of sources distributed solely in the near-field, amplitudes decay more rapidly than $1 / e^{\alpha r}$ (Tsai, 2011) and explaining the more rapid decay of the data points with the wrong model, would in this case result in a significant overestimation of the data points for long interstation distances. Ultimately, the resolution of our data does not allow a clear distinction between different radial source distribution models. However, if the assumed source distribution would be wrong, it would mainly change the absolute values of the obtained $\mathrm{Q}$, but not its variation as function of frequency. A uniform radial source distribution is additionally justified by the fact that all receivers are offshore.

In both Figures 9 and 10, the observed imaginary part of $\gamma(r)$ is close to zero, which points to a high degree of symmetry of the EGFs. This is an important indication that the azimuthal and temporal averaging of the available data successfully approximates an isotropic source distribution. We infer that it should be possible to obtain a good 1-D estimate of attenuation at the location of the array.

[Figure 11 about here.]

It is interesting to compare the data-fit of the damped Bessel functions to the fit achieved by Bessel functions that are not multiplied by an exponential term (i.e. MF is minimized with $\alpha$ set to 0). Fitting damped instead of pure Bessel functions (Figure 11) gives an overall decrease of MF for the vertical and DPG component of $58 \%$ and $17 \%$ respectively. 
The decrease is significantly lower for the DPG component as attenuation turns out to be lower for the acoustic mode waves.

The MF values associated with the best fit to $\gamma(r)$ are shown in Figure 12 and 13 as a function of frequency, together with their corresponding values for $A, c, \alpha$ and $Q$ for the vertical component and DPG component, respectively. All three variables in Equation 13 are essentially an average over the survey area because of the azimuthal averaging. At lower frequencies, the vertical-component phase velocity $c$ (Figure 12a) increases drastically, while above $0.30 \mathrm{~Hz}$, phase velocity is $\sim 700 \mathrm{~m} / \mathrm{s}$. For this reason, wavelengths of the best fitting functions are more than half the aperture of the array below $0.20 \mathrm{~Hz}$. Phase velocities for the DPG-component's frequency band are gently decreasing with increasing frequency; from just below $2100 \mathrm{~m} / \mathrm{s}$ to $\sim 1800 \mathrm{~m} / \mathrm{s}$. MF values are normalized with respect to values of A for a fair comparison between frequencies. For frequencies higher than $0.4 \mathrm{~Hz}, \mathrm{MF}$ increases significantly as $\gamma(r)$ is affected by the first-higher order mode Scholte waves.

We test the stability of the minima of the misfit function with a bootstrapping experiment: $90 \%$ of the total number of interstation-distance bins are randomly selected from the full set of bins; the same bin can be selected more than once. We calculate a new misfit function from the sample and determine the minimum. We iterate this process 100 times and end up with 100 estimates of $\alpha, A$ and $c$ per frequency: in the ideal case of a perfectly constrained solution, these estimates would all be equal.

[Figure 12 about here.]

[Figure 13 about here.]

Phase velocities prove to be well constrained as bootstrapping yields very little vari- 
ability; error bars are coinciding with median values and hence are hardly visible for both components (Figure 12a and 13a). The bootstrapping does yield some variability in $A$ and $\alpha$ values for both components. A slight trade-off between these two parameters can be observed if we examine the cost functions' $M F$ associated with the best fit to $\gamma(r)$ and fix the phase velocity $c$. For the lower frequencies in the vertical-component frequency band, i.e. towards $0.20 \mathrm{~Hz}$ and hence on the slope of the double-frequency microseism peak (Peterson, 1993), values for $A$ tend to be higher. This points to a lower power associated with the cross-terms which in turn indicates that the differences in power of different sources are low for these frequencies. The DPG-component frequency band shows the same for the higher frequencies.

The attenuation coefficient, $\alpha$, associated with the best fit of $M F$ to $\gamma(r)$ is presented in Figure 12c and 13c as a function of frequency and for the vertical and DPG component respectively. The quality factors associated with these decay values are shown in Figure 12e and 13e, respectively. Calculating the quality factors from $\alpha$ according to equation 12 requires knowledge of the Group velocity $U$. We determine $U$ by differentiating numerically our observed phase-velocity dispersion curve (Aki and Richards, 2002). The vertical component's group velocity is varying around $500 \mathrm{~m} / \mathrm{s}$ with an increasing trend, while the DPG component's group velocity is varying around $1400 \mathrm{~m} / \mathrm{s}$ with a decreasing trend (see Figure 12a and 13a respectively). These values are in agreement with the observed moveouts in Figure 3 .

Figure 13e shows that surface wave quality factor as a function of frequency oscillates around a mean value of $\sim 100$ for the DPG-component. As attenuation for this component turns out to be so low, a significant number of the bootstrapping samples yield an attenuation coefficient equal to zero for some frequencies; this is associated with error bars on 
the corresponding Q values reaching infinity. Variations become less severe with increasing frequency however. The vertical component Q (Figure 12e) oscillates around $\sim 20$, and decreases with decreasing frequency below $0.30 \mathrm{~Hz}$.

\section{COMPARISON WITH OTHER STUDIES}

Temporal and azimuthal averaging of spectral whitened data gives us realistic frequency dependent estimates of $Q$, and we infer that our method correctly extracts this information from the ambient seismic field. Our results show that the ambient seismic field carries significant information about the anelastic Earth structure. This has been shown before (Prieto et al., 2009; Lin et al., 2011; Lawrence and Prieto, 2011), but not for the spatial and temporal dimensions presented here.

The bulk of the Scholte and acoustic guided wave analysis performed is based on shallow water data, i.e. < $100 \mathrm{~m}$ (Klein et al., 2005; Zhou et al., 2009; Bohlen et al., 2004). The great water depth of this survey $(\sim 360 \mathrm{~m})$ causes a downward frequency shift of the modal transitions. Greater depths are able to accommodate lower frequency Scholte and acoustic wave modes. Forward modelling by Klein et al. (2005) indicated that an overlying water column of several hundred meters would be able to support acoustic guided waves below 1 Hz. This is exactly what we observe for this dataset. We will shortly discuss the obtained velocity and attenuation estimates for the respective components and the relation to the local geology. 


\section{DPG-component frequency band}

The phase velocities we obtain for the acoustic guided waves are, to a first order, in agreement with values found by others (Klein et al., 2005). Furthermore, the group velocity to be expected for the conditions of our survey is about $1478 \mathrm{~m} / \mathrm{s}$. We use Mackenzie's equation for the speed of sound in seawater (Mackenzie, 1981) using a depth of $360 \mathrm{~m}$, a temperature of $5^{\circ} \mathrm{C}$ and an average salinity of $36 \mathrm{ppt}$ (Berx and Hughes, 2009) to obtain this value. Our group velocity oscillates around this value although it is a bit lower for the higher frequencies.

The attenuation of acoustic guided waves for the frequencies considered here is still not fully understood and quality factors in plain seawater are unknown for the frequencies considered here (Jensen et al., 2000). Because the DPGs are measuring pressure fluctuations only $\sim 50 \mathrm{~cm}$ above the sea-bottom, it is unclear if or to what extent the estimated phase velocities and attenuation values need to be associated with sea-bed sediment estimates of these parameters. As the obtained attenuation coefficients are to a first order constant with frequency, we estimate the attenuation coefficient for the acoustic guided waves 0.00004 $\mathrm{Np} / \mathrm{m}$. This corresponds to a compressional wave quality factor of about 100 (see Figure 13). Future investigations focussing on similar frequencies and environments (water depth, temperature, salinity) have to confirm our findings.

Contrary to the fundamental-and first higher-mode Scholte waves, the slowness-frequency spectra of the vertical and transversal components do not show the acoustic guided waves (Bussat and Kugler, 2011). The radial component does record the acoustic guided waves however. This indeed suggests a possible interaction of these waves with the sea-bottom sediments. The very shallow sediments (upper $\sim 5 \mathrm{~m}$ ) act as a purely compressional medium 
because of the high water content (Jensen et al., 2000; Zhou et al., 2009). In general, the sound speed of these sediment sampling acoustic waves is only slightly higher than the speed of sound in seawater, with their ratio approaching 1 with increasing frequency (Klein et al., 2005). It would be interesting to estimate phase velocities and quality factors for the radial component in a similar fashion as the ones obtained for the DPG-component, and compare the parameters for the two different components. This is, however, beyond the scope of this investigation.

\section{Vertical component frequency band}

Anelastic attenuation of Scholte waves is poorly addressed for the low frequencies considered here. Broadhead et al. (1993) analyze Scholte wave attenuation at two sites off the coast of the Western US. For water depths of $2600 \mathrm{~m}$ and $3800 \mathrm{~m}$ they find average quality factors of about 30-40 for a frequency range of 0.3 to $6.0 \mathrm{~Hz}$. They expect, however, that the obtained Q's are biased to lower frequencies. The higher frequencies in the fundamental-mode Scholte wave frequency band give us surface wave quality factors around 20 (see Figure 12e) which is in good agreement with their values.

Nguyen et al. (2009) analyze shot recordings from one broad band OBS located at the Ninetyeast Ridge in the Indian Ocean. They find that a surface wave quality factor of 40 is required for the uppermost mushy layer to explain the observed amplitude decay of the Scholte waves with distance. The uppermost layer is relatively thin at the location they investigate and the frequencies of the observed Scholte waves are significantly higher. Nevertheless, their value is in agreement with our estimates. Other studies evaluating Scholte wave attenuation, end up with significantly higher estimates for surface wave quality 
factors (Bromirski et al., 1992; Nolet and Dorman, 1996).

\section{Relation to geology}

Relating the estimated $\mathrm{Q}$ values and phase velocities of the vertical component frequency band to the velocity inversion performed by Bussat and Kugler (2011), a first order translation to depth can be made. Phase velocity in the $0.30-0.40 \mathrm{~Hz}$ band with low values of $\sim 700 \mathrm{~m} / \mathrm{s}$ is sensitive up to depths of $1000 \mathrm{~m}$ below the sea-bottom, but most sensitive to the shallowest $\sim 150 \mathrm{~m}$. These shallow depth sediments therefore probably have an average $\mathrm{Q}$ value of about 20 . Phase velocity increases with decreasing frequency up to $1200 \mathrm{~m} / \mathrm{s}$ at 0.20 Hz. At this frequency, velocities are attributed to depths greater than $2000 \mathrm{~m}$ (Bussat and Kugler, 2011). The very low quality factors for the $0.20-0.30 \mathrm{~Hz}$ frequency band imply a highly attenuating body at greater depth.

[Figure 14 about here.]

Two exploration wells were drilled close to the center of the array (see Figure 2). Both wells penetrate to a depth of $\sim 2950$ m below the sea-bottom and their characteristics are listed by the Norwegian Petroleum Directorate (NPD, 2012). The main lithostratigraphic units shown in Figure 14 are based on the interpretation of the data of well 35/11-14 S. The depth of encounter of the various groups doesn't change significantly between this well and the other exploration well located within the array, i.e. well 35/11-13 (NPD, 2012); a maximum difference of $37 \mathrm{~m}$ is measured for the top of the Hordaland group while deeper groups show even less variation in their depth of encounter. Several other wells are located within $\sim 15 \mathrm{~km}$ of well 35/11-14 S; Their data demonstrate maximum lateral variations of 1000 meters of the main lithostratigraphic units' depth (NPD, 2012). 
The low quality factors we observe for the frequencies down to $0.20 \mathrm{~Hz}$ can potentially be attributed to the combined effect of chalk facies in the Shetland Group and shale facies in the Rogaland Group (NPD, 2012). In general, chalks are associated with lower quality factors than siliciclastic rocks or less porous carbonate rocks and shales are known to have low quality factors as well (Sherrif and Geldart, 1995). Reid et al. (2001) find shear wave quality factors of about 20 for the top 200 meters of the Shetland Group based on VSP datasets from the North Sea which is in good agreement with the low quality factors we find for the lower frequencies.

\section{Conclusions}

Despite a very limited acquisition geometry in both time and space, i.e. 16 continuously redeployed broad-band ocean-bottom seismometers recording synchronously for $\sim 24$ hours and a survey period of less than 2 weeks, we are able to extract stable EGFs from this data set. We demonstrate that the EGF's amplitudes show a larger decay with distance as would be expected from purely geometrical spreading. We find that the wavefield changes significantly over time and demonstrate that temporal and azimuthal averaging of such a wavefield provides us with a good representation of an isotropic wavefield. We obtain whitened complex coherency's real parts to which damped Bessel functions can be fitted reasonably well. Minimizing the misfit of these Bessel functions enables us to quantitatively determine the energy decay with distance. In this way we obtain very reasonable estimates for the quality factors of $\sim 100$ for seawater at $360 \mathrm{~m}$ depth and $\mathrm{Q}$ values of 25 for the sediments up to $1000 \mathrm{~m}$ depth, while greater depths show even lower quality factors. These quality factors are obtained by exploiting the ambient seismic field, which is unprecedented for an experiment of this scale and in this environment. 


\section{Acknowledgements}

We gratefully acknowledge support from the QUEST Initial Training Network funded within the EU Marie Curie actions programme. We are very grateful to Statoil for providing us with this data set. We thank Domenico Giardini for his constant support and encouragement. This study benefitted from interactions with Julie Verbeke, Roel Snieder, Peter Hanssen, Sacha Bussat and Clotaire Michel. We thank two anonymous reviewers and the Associate Editor for helpful comments. Figures were generated with the help of Generic Mapping Tools (Wessel and Smith, 1991) 


\section{REFERENCES}

Aki, K., 1957, Space and time spectra of stationary stochastic waves, with special reference to microtremors.: Bulletin of the Earthquake Research Institute, University of Tokyo, 35, 415-457.

Aki, K., and P. G. Richards, 2002, Quantitative Seismology, 2 ed.: University Science Books.

Asten, M. W., 2006, On bias and noise in passive seismic data from finite circular array data processed using SPAC methods: Geophysics, 71, V153-162.

Bensen, G. D., M. H. Ritzwoller, M. P. Barmin, A. L. Levshin, F. Lin, M. P. Moschetti, N. M. Shapiro, and Y. Yang, 2007, Processing seismic ambient noise data to obtain reliable broad-band surface wave dispersion measurements: Geophysical Journal International, 169, 1239-1260.

Berx, B., and S. L. Hughes, 2009, Climatology of surface and near-bed temperature and salinity on the north-west European continental shelf for 1971-2000: Continental Shelf Research, 29, $2286-2292$.

Bohlen, T., S. Kugler, G. Klein, and F. Theilen, 2004, 1.5D inversion of lateral variation of Scholte-wave dispersion: Geophysics, 69, 330-344.

Brenguier, F., N. M. Shapiro, M. Campillo, A. Nercessian, and V. Ferrazzini, 2007, 3D surface wave tomography of the Piton de la Fournaise volcano using seismic noise correlations: Geophysical Research Letters, 34, L02305.

Broadhead, M., H. Ali, and L. Bibee, 1993, Scholte wave attenuation estimates from two diverse test sites: OCEANS '93. Engineering in Harmony with Ocean. Proceedings, I114 $-\mathrm{I} 118$ vol.1.

Bromirski, P. D., L. N. Frazer, and F. K. Duennebier, 1992, Sediment shear Q from airgun 
OBS data: Geophysical Journal International, 110, 465-485.

Bussat, S., and S. Kugler, 2009, Recording noise - estimating shear-wave velocities: Feasibility of offshore ambient-noise surface-wave tomography (ANSWT) on a reservoir scale: SEG Technical Program Expanded Abstracts, 28, 1627-1631.

—_ 2011, Offshore ambient-noise surface-wave tomography above $0.1 \mathrm{~Hz}$ and its applications: The Leading Edge, 30, 514-524.

Campillo, M., and A. Paul, 2003, Long-range correlations in the diffuse seismic coda: Science, 299, 547-549.

Chapman, M., E. Liu, and X.-Y. Li, 2006, The influence of fluid-sensitive dispersion and attenuation on AVO analysis: Geophysical Journal International, 167, 89-105.

Chávez-García, F. J., M. Rodríguez, and W. R. Stephenson, 2005, An alternative approach to the SPAC analysis of microtremors: Exploiting stationarity of noise: Bulletin of the Seismological Society of America, 95, 277-293.

Claerbout, J. F., 1968, Synthesis of a layered medium from its acoustic transmission response: Geophysics, 33, 264-269.

Cox, C., T. Deaton, and S. Webb, 1984, A deep-sea differential pressure gauge: Journal of Atmospheric and Oceanic Technology, 1, 237.

Derode, A., E. Larose, M. Tanter, J. de Rosny, A. Tourin, M. Campillo, and M. Fink, 2003, Recovering the Green's function from field-field correlations in an open scattering medium (L): Journal of the Acoustical Society of America, 113, 2973-2976.

Draganov, D., K. Wapenaar, W. Mulder, J. Singer, and A. Verdel, 2007, Retrieval of reflections from seismic background-noise measurements: Geophysical Research Letters, 34, L04305.

Duvall, T. L., S. M. Jefferies, J. W. Harvey, and M. A. Pomerantz, 1993, Time-distance 
helioseismology: Nature, 362, 430-432.

Ekström, G., G. A. Abers, and S. C. Webb, 2009, Determination of surface-wave phase velocities across USArray from noise and Aki's spectral formulation: Geophysical Research Letters, 36, L18301.

Friedrich, A., F. Krüger, and K. Klinge, 1998, Ocean-generated microseismic noise located with the Gräfenberg array: Journal of Seismology, 2, no. 1, 47-64.

Goertz, A., B. Schechinger, B. Witten, M. Koerbe, and P. Krajewski, 2012, Extracting subsurface information from ambient seismic noise - a case study from germany: Geophysics, 77, KS13-KS31.

Hasselmann, K., 1963, A statistical analysis of the generation of microseisms: Rev. Geophys., 1, 177-210.

Jensen, F. B., W. A. Kuperman, M. B. Porter, and H. Schmidt, 2000, Computational ocean acoustics: Springer-Verlag.

Klein, G., T. Bohlen, F. Theilen, S. Kugler, and T. Forbriger, 2005, Acquisition and inversion of dispersive seismic waves in shallow marine environments: Marine Geophysical Research, 26, no. 2, 287-315.

Landés, M., F. Hubans, N. M. Shapiro, A. Paul, and M. Campillo, 2010, Origin of deep ocean microseisms by using teleseismic body waves: Journal of Geophysical Research, 115, B05302.

Larose, E., L. Margerin, A. Derode, B. van Tiggelen, M. Campillo, N. Shapiro, A. Paul, L. Stehly, and M. Tanter, 2006, Correlation of random wavefields: An interdisciplinary review: Geophysics, 71, SI11-21.

Lawrence, J. F., and G. A. Prieto, 2011, Attenuation tomography of the western United States from ambient seismic noise: Journal of Geophysical Research, 116, B06302. 
Lin, F.-C., M. H. Ritzwoller, and W. Shen, 2011, On the reliability of attenuation measurements from ambient noise cross-correlations: Geophysical Research Letters, 38, L11303.

Lobkis, O. I., and R. L. Weaver, 2001, On the emergence of the Green's function in the correlations of a diffuse field: The Journal of the Acoustical Society of America, 110, $3011-3017$.

Longuet-Higgins, M. S., 1950, A theory of the origin of microseisms: Philosophical Transactions of the Royal Society of London. Series A, Mathematical and Physical Sciences, 243, 1-35.

Mackenzie, K. V., 1981, Nine term equation for sound speed in the oceans: Journal of the Acoustical Society of America, 70, 807-812.

McNamara, D. E., and R. P. Buland, 2004, Ambient noise levels in the continental United States: Bulletin of the Seismological Society of America, 94, 1517-1527.

Nguyen, X., T. Dahm, and I. Grevemeyer, 2009, Inversion of Scholte wave dispersion and waveform modeling for shallow structure of the Ninetyeast Ridge: Journal of Seismology, 13, no. 4, 543-559.

Nolet, G., and L. M. Dorman, 1996, Waveform analysis of Scholte modes in ocean sediment layers: Geophysical Journal International, 125, 385-396.

NPD, 2012, Norwegian petroleum directorate factpages, http://factpages.npd.no/factpages/default.aspx, accessed 19 March, 2012.

Okada, H., 2003, The microtremor survey method: Society of Exploration Geophysicists. Geophysical Monograph, No. 12.

Olofsson, B., 2010, Marine ambient seismic noise in the frequency range 1-10 Hz: The Leading Edge, 29, 418-435.

Peterson, J., 1993, Observation and modeling of seismic background noise: U.S. Geological 
Survey Open-File Report 93-322.

Poli, P., H. A. Pedersen, M. Campillo, and the POLENET/LAPNET Working Group, 2012, Emergence of body waves from cross-correlation of short period seismic noise: Geophysical Journal International, 188, 549-558.

Prieto, G. A., J. F. Lawrence, and G. C. Beroza, 2009, Anelastic Earth structure from the coherency of the ambient seismic field: Journal of Geophysical Research, 114, B07303.

Reid, F. J. L., P. H. Nguyen, C. MacBeth, and R. A. Clark, 2001, Q estimates from North Sea VSPs: SEG Technical Program Expanded Abstracts, 20, 440-443.

Rickett, J., and J. Claerbout, 1999, Acoustic daylight imaging via spectral factorization; helioseismology and reservoir monitoring: The Leading Edge, 18, 957-960.

Ringler, A. T., and C. R. Hutt, 2010, Self-noise models of seismic instruments: Seismological Research Letters, 81, 972-983.

Roux, P., K. G. Sabra, P. Gerstoft, W. A. Kuperman, and M. C. Fehler, 2005, P-waves from cross-correlation of seismic noise: Geophysical Research Letters, 32, L19303.

Scholte, J., 1943, Over het verband tussen zeegolven en microseismen, I and II: Verslag Nederlands Akademie van Wetenschappen, 52, 669-683.

Seats, K. J., J. F. Lawrence, and G. A. Prieto, 2012, Improved ambient noise correlation functions using Welch's method: Geophysical Journal International, 188, 513-523.

Shapiro, N. M., and M. Campillo, 2004, Emergence of broadband Rayleigh waves from correlations of the ambient seismic noise: Geophysical Research Letters, 31, L07614.

Shapiro, N. M., M. Campillo, L. Stehly, and M. H. Ritzwoller, 2005, High-resolution surfacewave tomography from ambient seismic noise: Science, 307, 1615-1618.

Sherrif, R. E., and L. P. Geldart, 1995, Exploration seismology, 2 ed.: Cambridge University Press. 
Snieder, R., 2004, Extracting the Green's function from the correlation of coda waves: a derivation based on stationary phase: Physical Review E, 69, no. 4, 046610.

Snieder, R., K. Wapenaar, and U. Wegler, 2007, Unified Green's function retrieval by crosscorrelation; connection with energy principles: Physycial Review E, 75, 036103.

Stehly, L., M. Campillo, and N. M. Shapiro, 2006, A study of the seismic noise from its long-range correlation properties: Journal of Geophysical Research, 111, B10306.

Tsai, V. C., 2009, On establishing the accuracy of noise tomography travel-time measurements in a realistic medium: Geophysical Journal International, 178, 1555-1564.

—, 2011 , Understanding the amplitudes of noise correlation measurements: Journal of Geophysical Research, 116, B09311.

Tsai, V. C., and M. P. Moschetti, 2010, An explicit relationship between time-domain noise correlation and spatial autocorrelation (SPAC) results: Geophysical Journal International, 182, 454-460.

Wapenaar, C., D. Draganav, R. Snieder, X. Campman, and A. Verdel, 2010, Tutorial on seismic interferometry: Part 1 - basic principles and applications: Geophysics, 75, 75A195 $-75 \mathrm{~A} 209$.

Wapenaar, K., 2004, Retrieving the elastodynamic Green's function of an arbitrary inhomogeneous medium by cross correlation: Physical Review Letters, 93, no. 25, 254301.

Wessel, P., and W. H. F. Smith, 1991, Free software helps map and display data: EOS Transactions, $\mathbf{7 2}, 441-446$.

Yang, Y., and M. H. Ritzwoller, 2008, Characteristics of ambient seismic noise as a source for surface wave tomography: Geochemistry, Geophysics, Geosystems, 9, Q02008.

Yang, Y., M. H. Ritzwoller, A. L. Levshin, and N. M. Shapiro, 2007, Ambient noise Rayleigh wave tomography across Europe: Geophysical Journal International, 168, 259-274. 
Yokoi, T., and S. Margaryan, 2008, Consistency of the spatial autocorrelation method with seismic interferometry and its consequence: Geophysical Prospecting, 56, 435-451.

Zhang, J., P. Gerstoft, and P. M. Shearer, 2009, High-frequency P-wave seismic noise driven by ocean winds: Geophysical Research Letters, 36, L09302.

Zhou, J.-X., X.-Z. Zhang, and D. P. Knobles, 2009, Low-frequency geoacoustic model for the effective properties of sandy seabottoms: Journal of the Acoustical Society of America, 125, 2847-2866. 


\section{LIST OF FIGURES}

1 The configuration of the survey. The stations are shown by the color-filled circles. The colors represents the total recording time of the station. Two black circles mark the "reference" stations that were recording continuously for a period of over 12 days. The locations of two exploration wells are represented by red stars. . . . . . . . . . . . . . . . . . .

2 The number of synchronous time windows (top) and station couples (bottom) per interstation distance. The bin size is $100 \mathrm{~m}$. . . . . . . . . . . . .

3 GFG of the vertical component for 0.2 to $0.4 \mathrm{~Hz}$ (a) and of the DPGcomponent for 1.0 to $2.0 \mathrm{~Hz}(\mathrm{~b})$. Only crosscorrelations based on synchronous recordings of more than 4 hours, interstation distances of more than $3.2 \mathrm{~km}$ and $S N R>4$ are displayed. . . . . . . . . . . . . . . .

4 Polar plots show the difference between the amplitude of causal and anticausal parts of the EGFs, for four example days of recording, with each panel corresponding to one day. We only take into account here vertical-component crosscorrelations based on synchronous recordings $>4$ hours, interstation distances $>3.2 \mathrm{~km}$ and $a S N R>4$, and normalize each of them individually to a maximum amplitude of 1 . Station couples that fulfilled these criteria are connected by lines in the inset maps. Each dot in a polar plot corresponds to one station couple, its angular coordinate coinciding with the station-couple back azimuth, and it's distance from the center of the plot proportional to the difference between causal and anti-causal amplitude. Red dots correspond to the crosscorrelations shown in Figure 5. . . . . . . . . . . . . . .

$5 \quad$ EGF with the lowest (a) and highest (b) amplitude difference over the course of the four days evaluated. . . . . . . . . . . . . . . . . . . . .

6 Amplitude of the Green's function with distance along two station lines of the causal (right) and anti-causal (left) parts. The stations for which recordings are crosscorrelated, are shown in the inset at the bottom. The green EGF amplitudes are based on 30 hours of synchronous recordings and the red amplitudes on 12 hours of synchronous recordings. The fitted curves are color coded correspondingly. The solid curves behave with $r$ as $(a / \sqrt{r}) \times e^{-\alpha r}$ while the dashed curves don't account for attenuation and follow an $a / \sqrt{r}$ trend. Amplitudes are normalized with respect to the value of the attenuating model (solid lines) at $3.2 \mathrm{~km}$ that fits the data best, i.e a. Normalization is performed for each line individually. . . . . . . . . . . . . . . . .

$7 \quad$ Power spectral densities for stations P01 ( $a$ and $c$ ) and B01 ( $b$ and $d$ ) during daytime ( $a$ and $b$ ) and nighttime ( $c$ and d) for two hours of recording. Each plot shows 120 PSDs of 60 seconds windows in grey, while the thick solid black line gives the mean PSD for the two hour period. The dashed line denotes the low-noise model of Peterson (1993). The daytime PSDs in the left column are computed from recordings on 2007-04-27, 14:00:00-16:00:00 UTC, while the nighttime PSDs in the right column are calculated from recordings on 2007-04-28, 00:00:00-02:00:00 UTC. . . . . . . . . . . . 
8 Real part of the whitened complex coherency for interstation distances 50012000 meters. Vertical component results are shown for 0.20-0.40 Hz (a) and $D P G$-component real parts for 1.50-2.20 Hz (b). The cross sections associated with dashed black lines at $0.25 \mathrm{~Hz}$ and $2.00 \mathrm{~Hz}$ are shown in Figure 9 and 10 respectively. The triangles on the scale bar indicate that the maximum and minimum values are off-scale. . . . . . . . . . . . . . . . . .

9 Observed real (green dots) and imaginary (red dots) parts of the binned, whitened complex coherency $\gamma(r)$, for the vertical component at a frequency of $0.25 \mathrm{~Hz}$, compared to the corresponding best fitting Bessel function, with (solid black line) and without (dashed black line) attenuation factor. The two histograms on top show the data coverage as a function of interstation distance. The red histogram indicates the number of time windows while the yellow histogram gives the number of station couples. . . . . . . . . . . .

10 Same as Figure 9, but for the DPG component at $2.0 \mathrm{~Hz} \ldots \ldots$. . . . . .

11 Difference between the (non-damped) Bessel functions and the real parts of $\gamma(r, \omega)(a)$ and the damped Bessel functions that best fit the real parts $\gamma(r, \omega)$ and these real parts itself (b). Whitened complex coherencies and models for the 0.20-0.40 Hz vertical component data are shown. The models as well as the real parts of $\gamma(r, \omega)$ are normalized with respect to the proportionality factor $A$ before differences are calculated. The difference between the model and the data in (a) can be associated with attenuation due to the medium. The cross sections associated with dashed black lines at $0.25 \mathrm{~Hz}$ and $2.00 \mathrm{~Hz}$ are shown in Figure 9 and 10 respectively. The triangles on the color bar indicate that the maximum and minimum values are off-scale. . . . . . . . .

12 Bootstrapping results as a function of frequency for the vertical component's fundamental-mode Scholte waves. Phase velocity (a), vertical offset at $r=$ 0, A (b), $\alpha(c)$, normalized MF (d) and quality factor (e) are shown from top to bottom, respectively. The dots denote the median of the calculated values. Lower and upper error bars correspond to the $15.9^{\text {th }}$ percentile and $84.1^{\text {th }}$ percentile respectively, i.e. the equivalent of 1 standard deviation for a Gaussian distribution. The solid and dashed curves in a are the phase and group velocity respectively. . . . . . . . . . . . . . . . . . .

13 Same as Figure 12, but for the DPG component and frequencies between 1.50

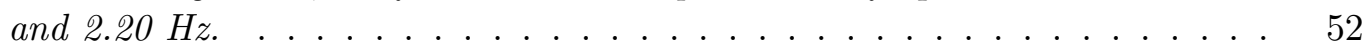

14 Geological groups as they were encountered while drilling well 35/11-14 S. . 


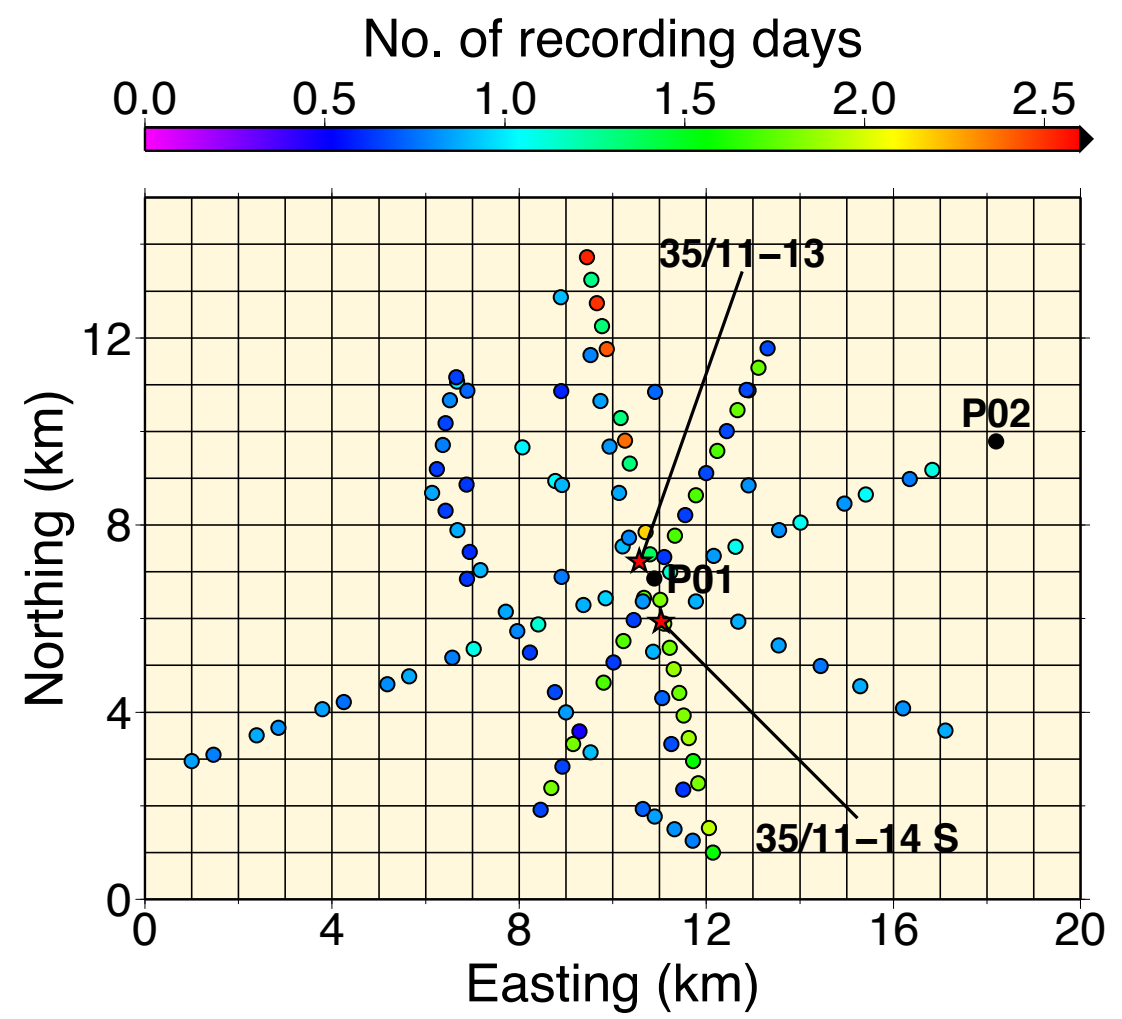

Figure 1: The configuration of the survey. The stations are shown by the color-filled circles. The colors represents the total recording time of the station. Two black circles mark the "reference" stations that were recording continuously for a period of over 12 days. The locations of two exploration wells are represented by red stars. 


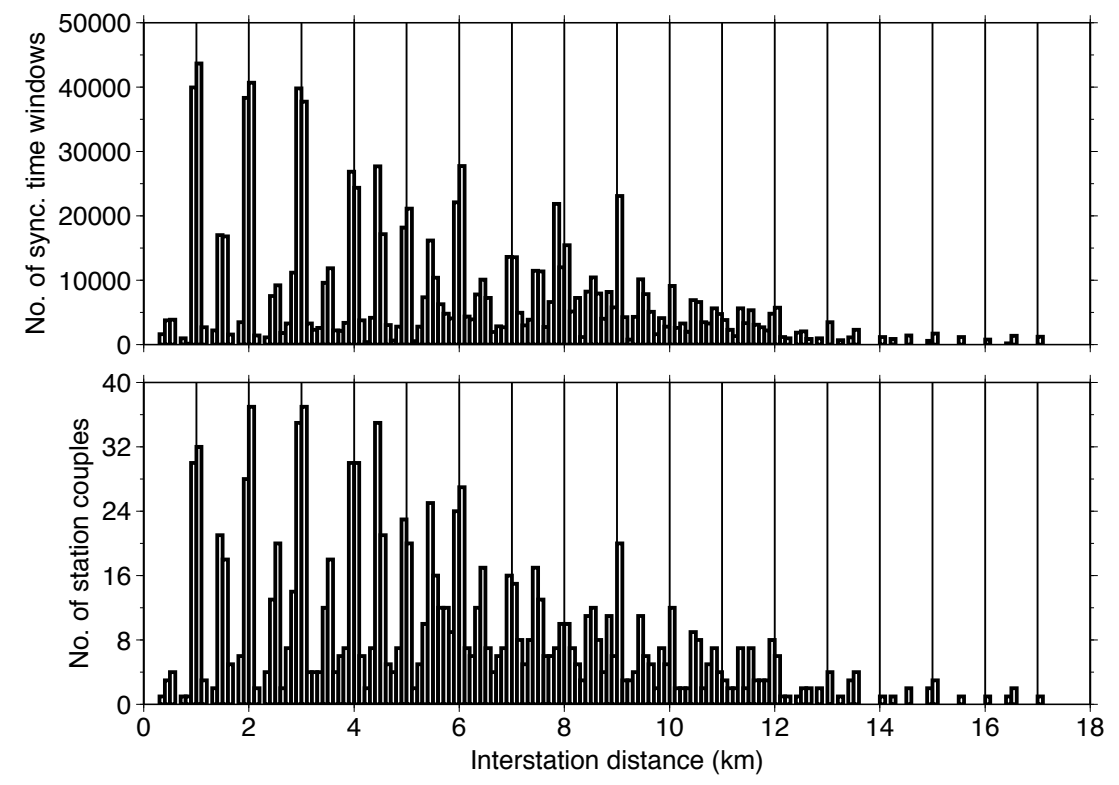

Figure 2: The number of synchronous time windows (top) and station couples (bottom) per interstation distance. The bin size is $100 \mathrm{~m}$. 

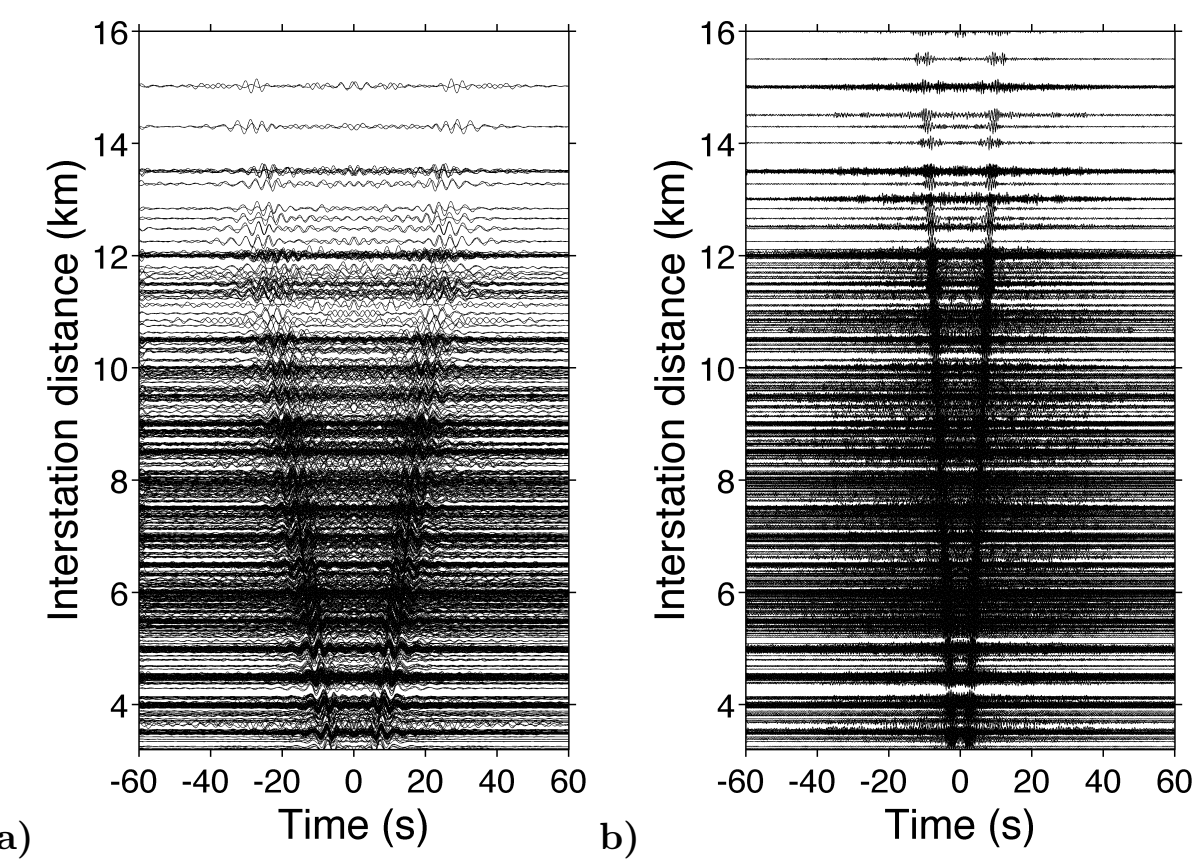

Figure 3: GFG of the vertical component for 0.2 to $0.4 \mathrm{~Hz}(\mathrm{a})$ and of the DPG-component for 1.0 to $2.0 \mathrm{~Hz}(b)$. Only crosscorrelations based on synchronous recordings of more than 4 hours, interstation distances of more than $3.2 \mathrm{~km}$ and $S N R>4$ are displayed. 


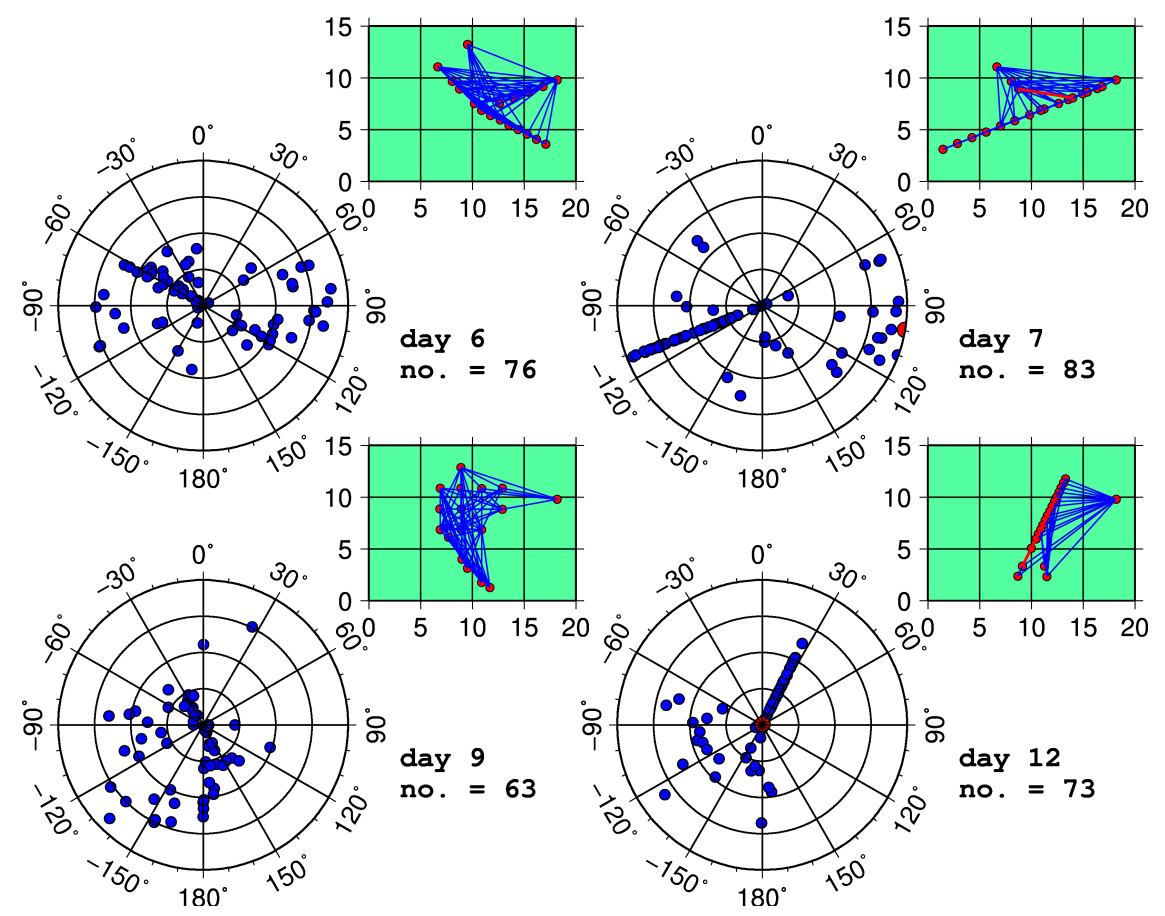

Figure 4: Polar plots show the difference between the amplitude of causal and anti-causal parts of the EGFs, for four example days of recording, with each panel corresponding to one day. We only take into account here vertical-component crosscorrelations based on synchronous recordings $>4$ hours, interstation distances $>3.2 \mathrm{~km}$ and $a S N R>4$, and normalize each of them individually to a maximum amplitude of 1. Station couples that fulfilled these criteria are connected by lines in the inset maps. Each dot in a polar plot corresponds to one station couple, its angular coordinate coinciding with the station-couple back azimuth, and it's distance from the center of the plot proportional to the difference between causal and anti-causal amplitude. Red dots correspond to the crosscorrelations shown in Figure 5 


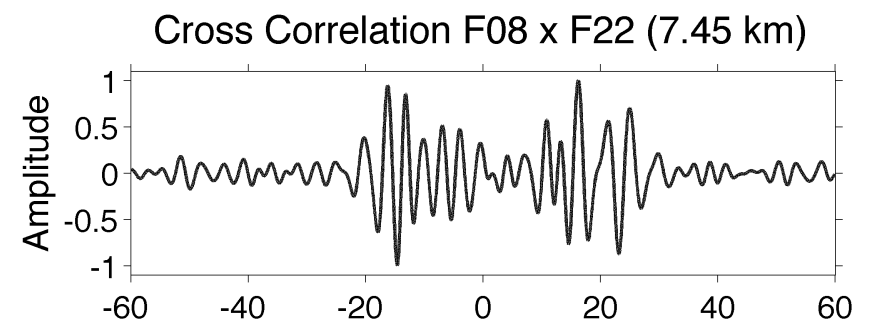

a)

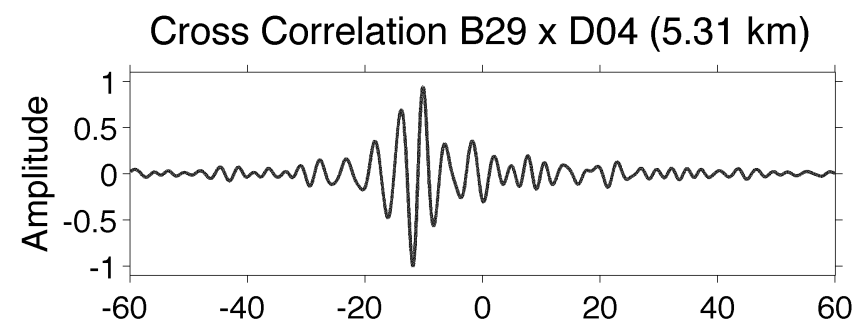

b)

Figure 5: EGF with the lowest (a) and highest (b) amplitude difference over the course of the four days evaluated. 


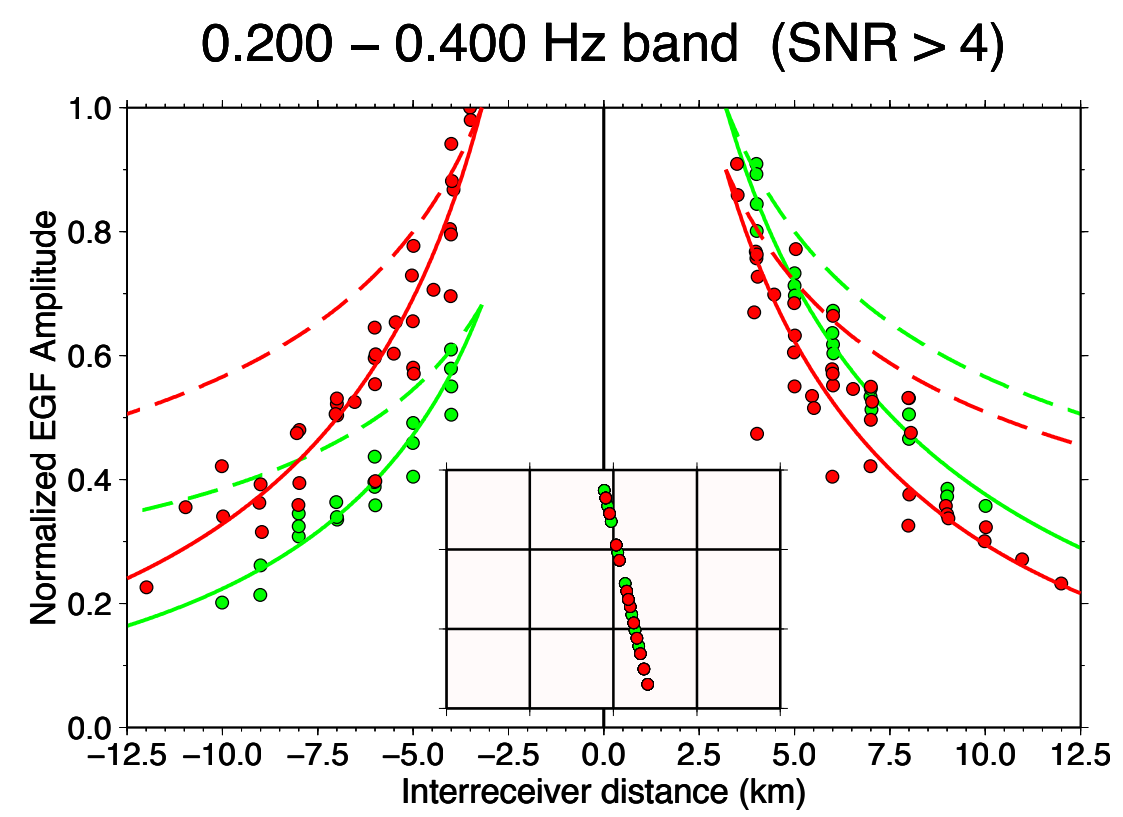

Figure 6: Amplitude of the Green's function with distance along two station lines of the causal (right) and anti-causal (left) parts. The stations for which recordings are crosscorrelated, are shown in the inset at the bottom. The green EGF amplitudes are based on 30 hours of synchronous recordings and the red amplitudes on 12 hours of synchronous recordings. The fitted curves are color coded correspondingly. The solid curves behave with $r$ as $(a / \sqrt{r}) \times e^{-\alpha r}$ while the dashed curves don't account for attenuation and follow an $a / \sqrt{r}$ trend. Amplitudes are normalized with respect to the value of the attenuating model (solid lines) at $3.2 \mathrm{~km}$ that fits the data best, i.e a. Normalization is performed for each line individually. 

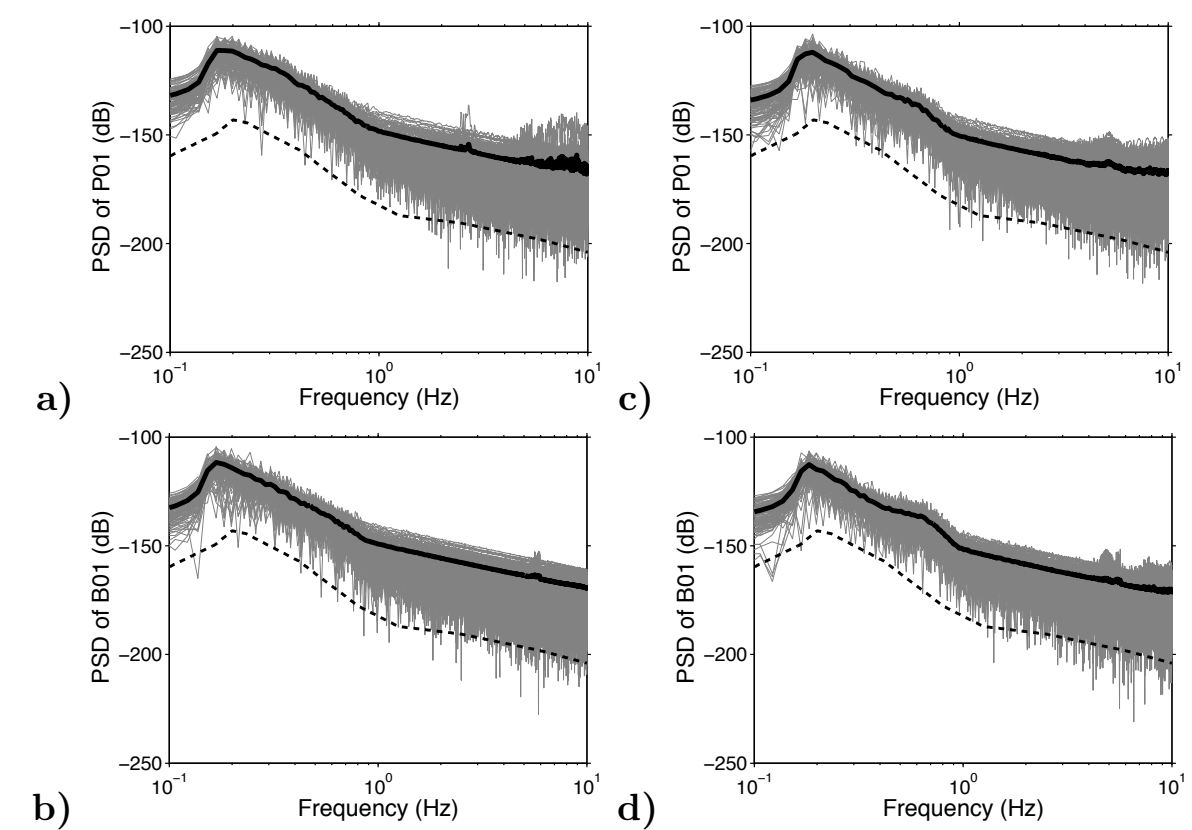

c)

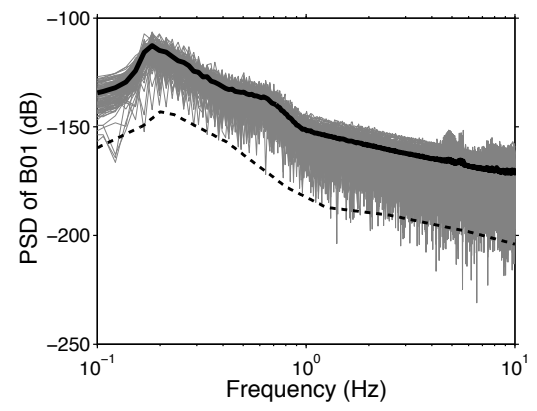

Figure 7: Power spectral densities for stations P01 ( $a$ and $c$ ) and B01 ( $b$ and $d$ ) during daytime ( $a$ and $b$ ) and nighttime ( $c$ and $d$ ) for two hours of recording. Each plot shows 120 PSDs of 60 seconds windows in grey, while the thick solid black line gives the mean PSD for the two hour period. The dashed line denotes the low-noise model of Peterson (1993). The daytime PSDs in the left column are computed from recordings on 2007-04-27, 14:00:00-16:00:00 UTC, while the nighttime PSDs in the right column are calculated from recordings on 2007-04-28, 00:00:00-02:00:00 UTC. 
a)

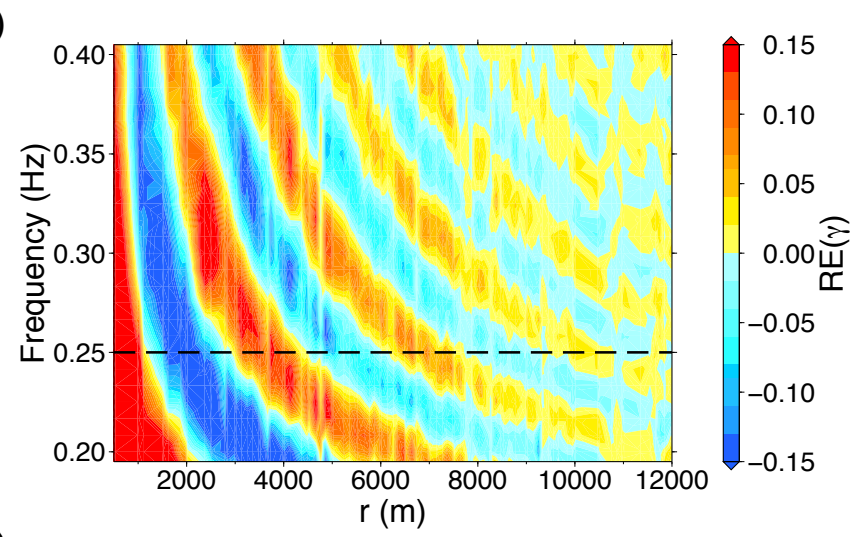

b)

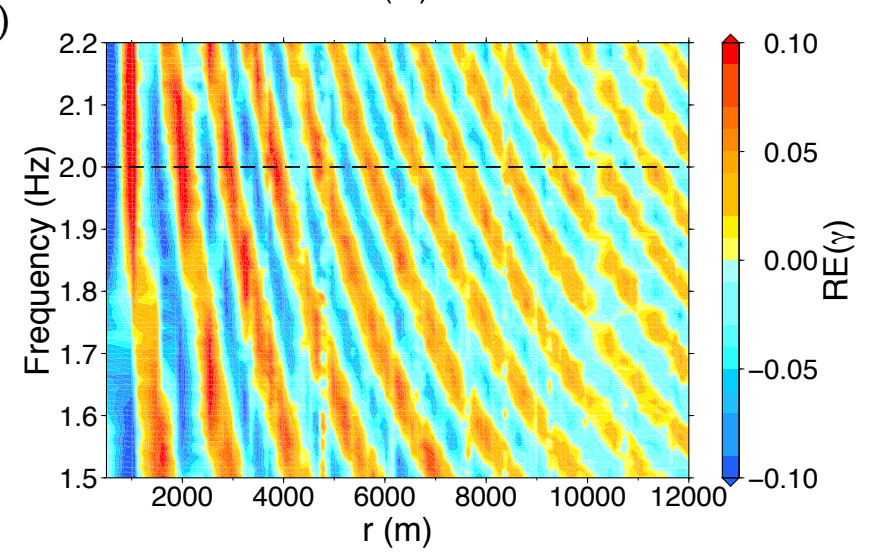

Figure 8: Real part of the whitened complex coherency for interstation distances 500-12000 meters. Vertical component results are shown for 0.20-0.40 Hz (a) and DPG-component real parts for 1.50-2.20 Hz (b). The cross sections associated with dashed black lines at 0.25 $\mathrm{Hz}$ and $2.00 \mathrm{~Hz}$ are shown in Figure 9 and 10 respectively. The triangles on the scale bar indicate that the maximum and minimum values are off-scale. 

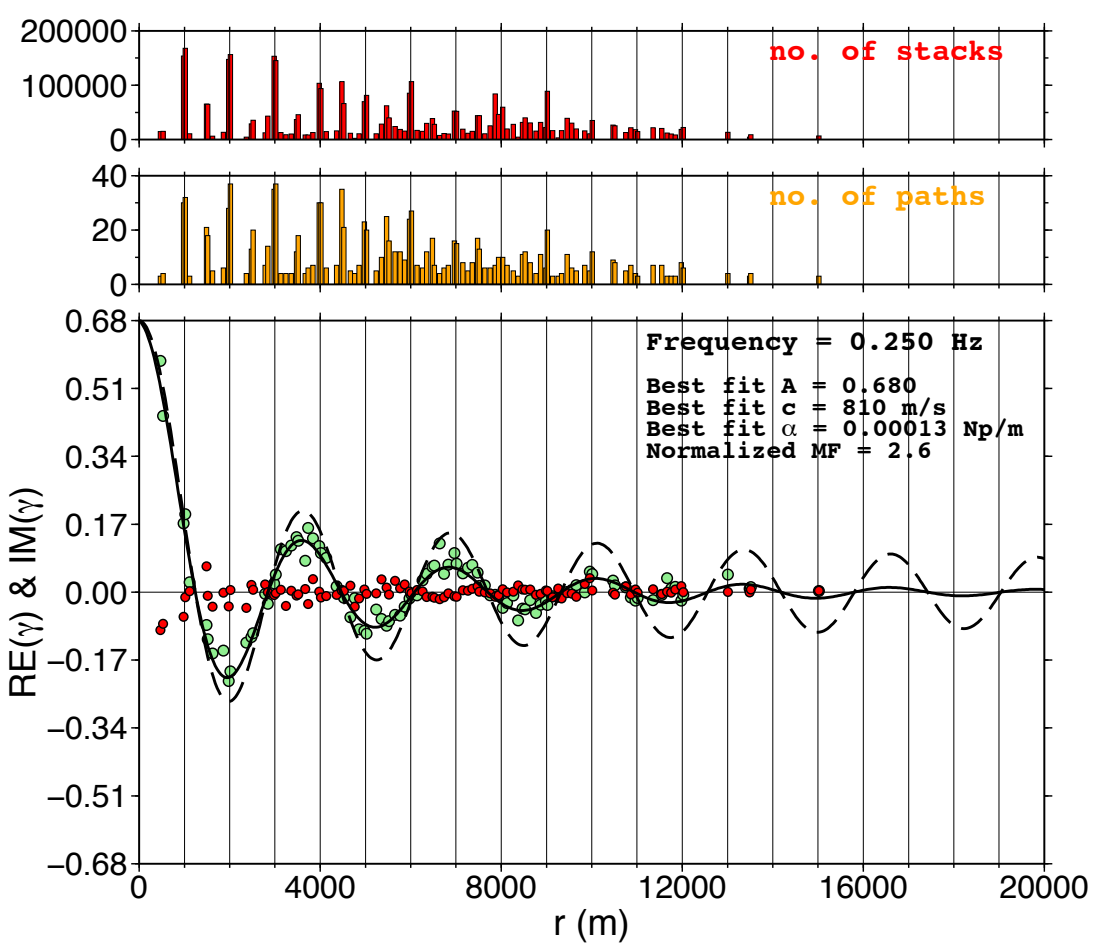

Figure 9: Observed real (green dots) and imaginary (red dots) parts of the binned, whitened complex coherency $\gamma(r)$, for the vertical component at a frequency of $0.25 \mathrm{~Hz}$, compared to the corresponding best fitting Bessel function, with (solid black line) and without (dashed black line) attenuation factor. The two histograms on top show the data coverage as a function of interstation distance. The red histogram indicates the number of time windows while the yellow histogram gives the number of station couples. 


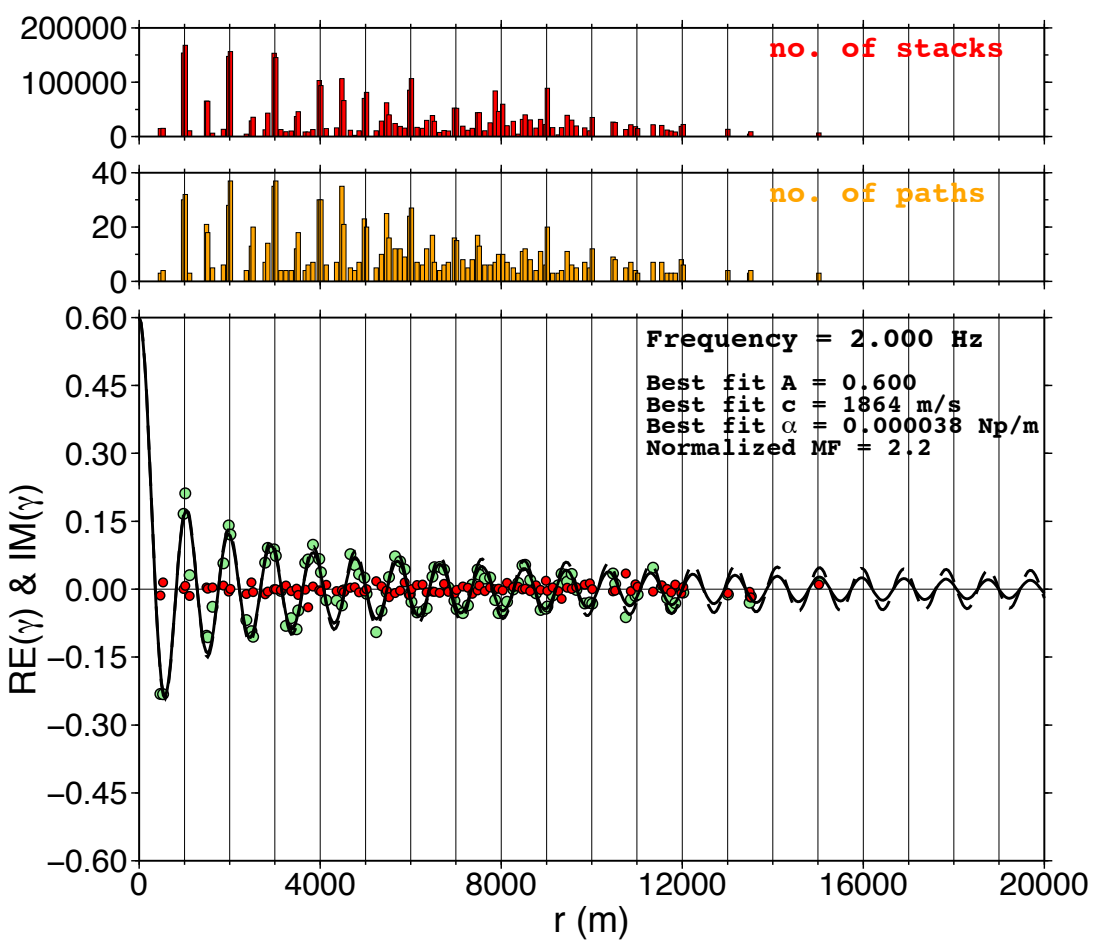

Figure 10: Same as Figure 9, but for the DPG component at $2.0 \mathrm{~Hz}$ 

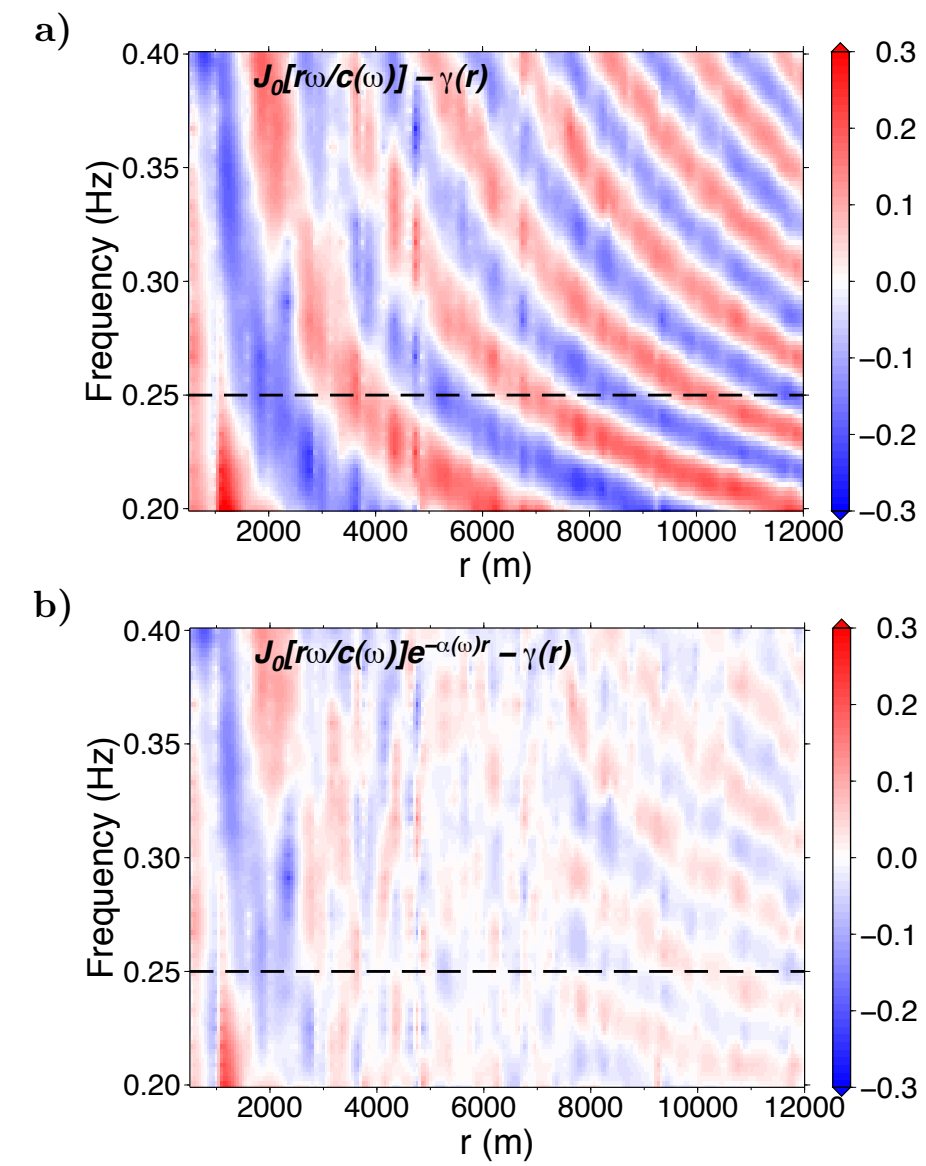

Figure 11: Difference between the (non-damped) Bessel functions and the real parts of $\gamma(r, \omega)$ (a) and the damped Bessel functions that best fit the real parts $\gamma(r, \omega)$ and these real parts itself (b). Whitened complex coherencies and models for the 0.20-0.40 Hz vertical component data are shown. The models as well as the real parts of $\gamma(r, \omega)$ are normalized with respect to the proportionality factor A before differences are calculated. The difference between the model and the data in (a) can be associated with attenuation due to the medium. The cross sections associated with dashed black lines at $0.25 \mathrm{~Hz}$ and $2.00 \mathrm{~Hz}$ are shown in Figure 9 and 10 respectively. The triangles on the color bar indicate that the maximum and minimum values are off-scale. 


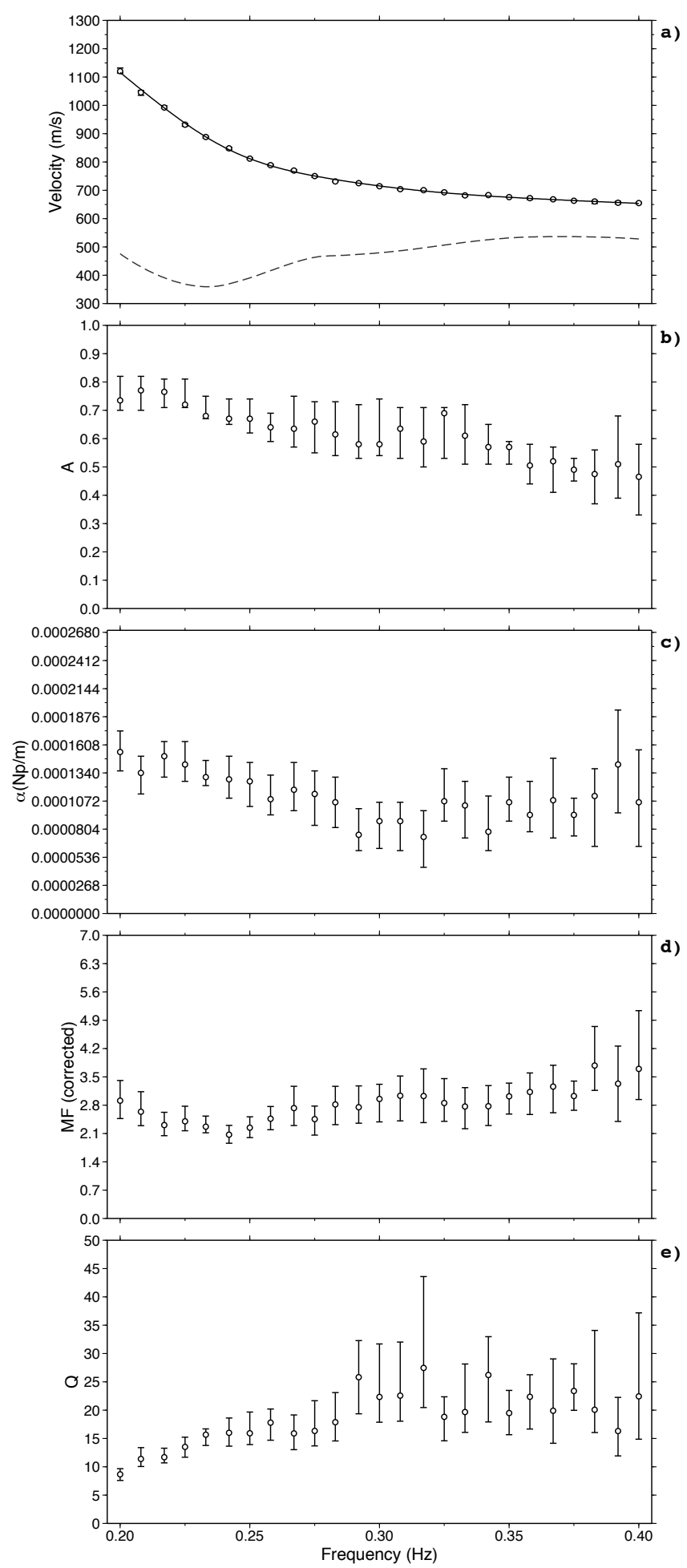

Figure 12: Bootstrapping results as a function of frequency for the vertical component's fundamental-mode Scholte waves. Phase velocity (a), vertical offset at $r=0, A(b), \alpha(c)$, normalized $M F(d)$ and quality factor (e) are shown from top to bottom, respectively. The dots denote the median of the calculated values. Lower and upper error bars correspond to the $15.9^{\text {th }}$ percentile and $84.1^{\text {th }}$ percentile respectively, i.e. the equivalent of 1 standard deviation for a Gaussian distribution. The solid and dashed curves in a are the phase and group velocity respectively. 


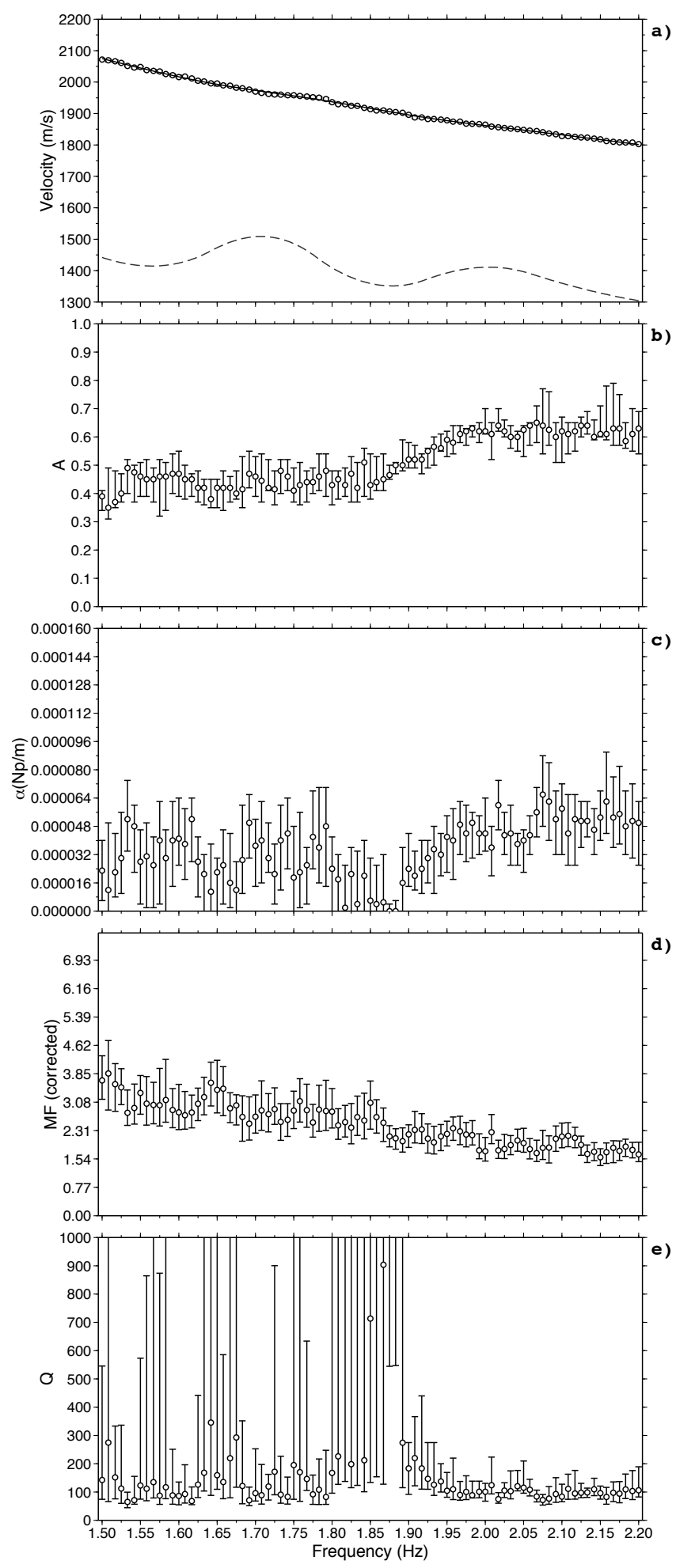

Figure 13: Same as Figure 12, but for the DPG component and frequencies between 1.50 and $2.20 \mathrm{~Hz}$. 


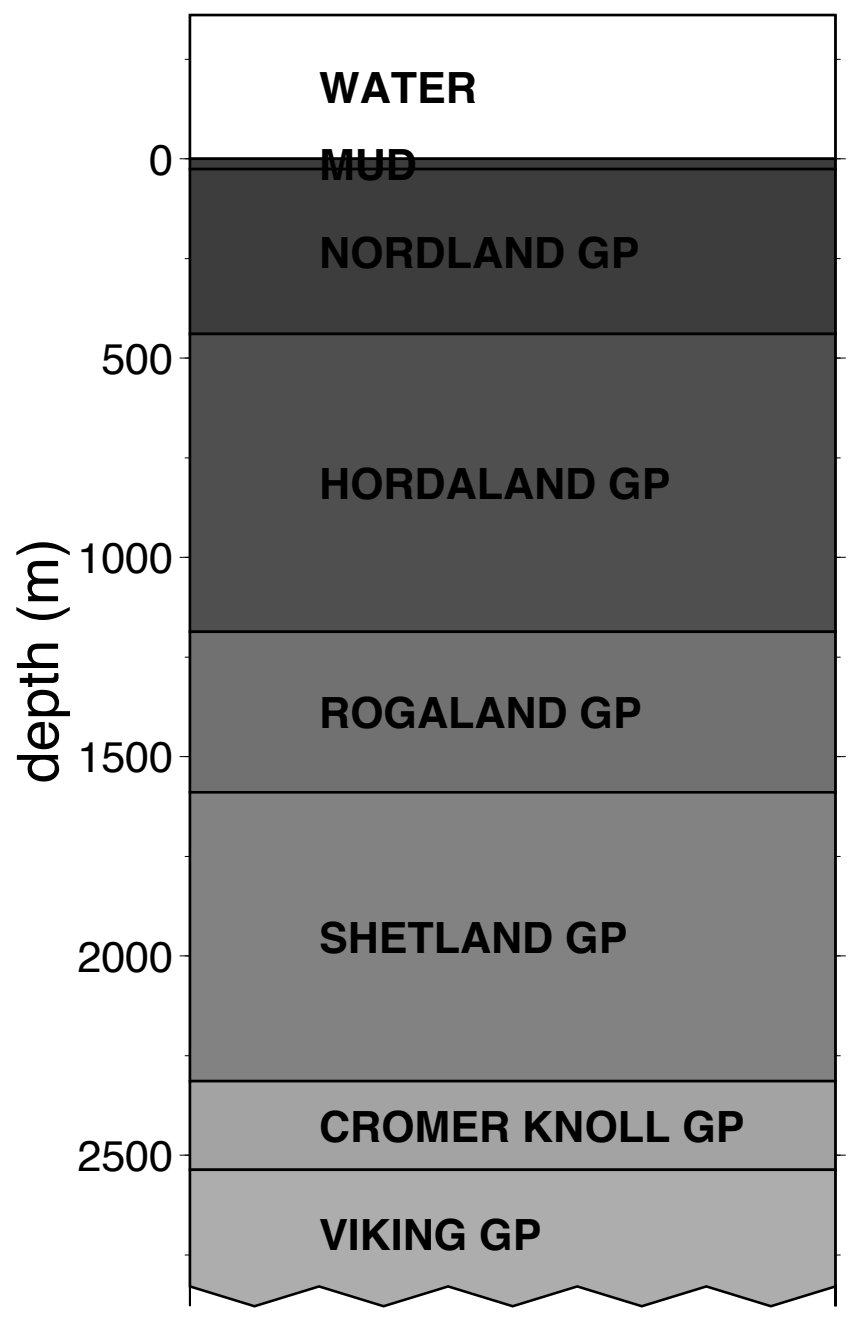

Figure 14: Geological groups as they were encountered while drilling well 35/11-14 S. 